\title{
EAl Endorsed Transactions

\section{Power System Flexibility Metrics Evaluation and Power Ramping Analysis for High Variable Renewable Generation Shares}

\author{
M. Saber Eltohamy ${ }^{1, *}$, M. Said Abdel Moteleb ${ }^{2}$, Hossam Talaat $^{3}$, S. Fouad Mekhemer ${ }^{3}$ and Walid \\ $\mathrm{Omran}^{4}$ \\ ${ }^{1}$ Research Assistant in Department of Power Electronics and Energy Conversion, Electronics Research Institute \\ (postgraduate student at Ain Shams university), Joseph Tito St, Huckstep, Qism El-Nozha, Cairo Governorate, Egypt,12622 \\ ${ }^{2}$ Professor in Department of Power Electronics and Energy Conversion, Electronics Research Institute, Joseph Tito St, \\ Huckstep, Qism El-Nozha, Cairo Governorate, Egypt, 12622 \\ ${ }^{3}$ Professor in Department of Electrical Engineering, Future University in Egypt, Cairo, Egypt, 11835 \\ ${ }^{4}$ Associate Professor in Department of Electrical Engineering, Future University in Egypt ( on leave from Ain Shams \\ University), Cairo, Egypt, 11835
}

\section{Abstract}

The increased penetrations from variable renewable generation (VRG), such as solar and wind, into power systems, growing the flexibility requirements for managing the uncertainty and variability of output power. These flexibility requirements can be achieved by many flexibility options. However, quantifying the flexibility available and required in a power system is a complicated problem. The paper reviews different flexibility metrics that measure the flexibility available from each conventional generator and that measure the flexibility available and needed by a power system at either planning and operational stages. Due to the dynamics of power systems, no flexibility metric has been taken as a standard. Accordingly, for high variable renewable generation shares, it is necessary to have a deeper understanding of power ramping scale as well as times when collective ramp events are most likely to occur. The paper introduces a method for power ramping analysis that will be demonstrated by analysing the output power of aggregated Belgian wind farms. In addition, the ramp characteristic indicators have been proposed for comparing the ramping behaviour of VRG in different years or between different countries.

Keywords: Power System Flexibility, Flexibility Metrics, Variable Renewable Generation (VRG), Power Ramping Analysis.

Received on 14 July 2019, accepted on 13 June 2020, published on 16 June 2020

Copyright ( $) 2020$ M. Saber Eltohamy et al., licensed to EAI. This is an open access article distributed under the terms of the Creative Commons Attribution licence (http://creativecommons.org/licenses/by/3.0/), which permits unlimited use, distribution and reproduction in any medium so long as the original work is properly cited.

doi: 10.4108/eai.13-7-2018.165282

"Corresponding author. Email:mohammed_saber@eri.sci.eg

\section{Nomenclature}

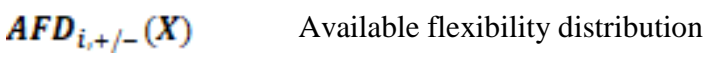

Contingency $y_{t}$ The contingency reserve requirement
D

$d_{n}$ Flex $_{r}$, FLEX $_{A}$

flex $_{\text {tris }} i_{i t}$

flex $_{\text {SYSTEM } i_{1}+1-}$
Annual electricity demand

The total number of historical day readings Flexibility index of the individual generator (r) and the entire system flexibility Available upward flexibility.

System flexibility time series. 


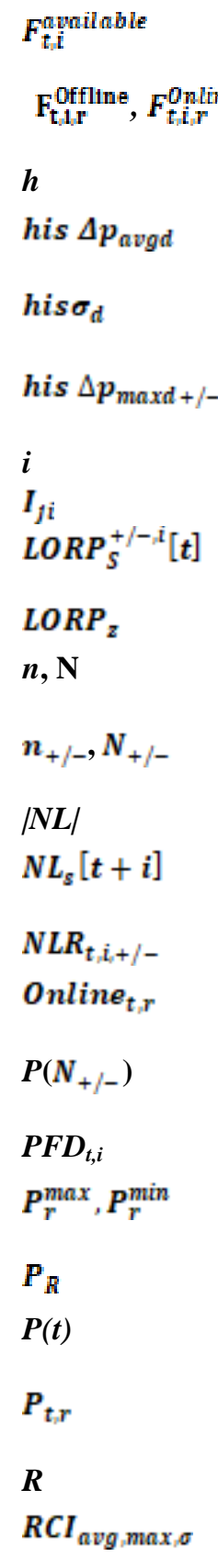

$R C I_{\text {ramping range }}$

$R C_{z}$

$R F_{t i s}$

$R R_{r_{1}+-}$

$S$

$S_{r}$

$t$

$v, V$

$W_{f}$
The available flexibility from online and offline power resources.

The available flexibility from an offline and online resource $r$ at observation time $t$.

The length of the power curve time series.

The average value of historical daily power ramps

The standard deviation of historical daily power ramps

The maximum upward and downward value of historical daily power ramps

The time interval

The normalized value of indicator $j$

lack of ramp probability in the system in both directions

Lack of Ramp Probability for zone

A counter for historical readings and the total number

A counter for upward and downward ramps in historical readings and the total number

Length of the net load time series

The expected system net load in the next period after time step i.

Up and down net load ramping time series

A binary variable $\{0,1\}$ that indicates the online resource state at observation time $(t)$.

Probability of occurrence of a ramp event with a certain direction

Period of flexibility deficit

The maximum and minimum output power from generator or power resource $r$

\section{Rated Power}

The output power at time $t$ from a spatial wind/PV unit

The output power of resource $r$ at observation time $\mathrm{t}$

Total number of power resources

Ramp characteristic indicators for the average, maximum and standard deviation of power ramps

Ramp characteristic indicator for the ramping range

Zone ramp up capability

The realized flexibility for scenario $s$ at observation time $t$

The upward and downward ramp rate of resource $r$

The VRG output power scenario

Resource start-up time

The Observation time

The largest and the target variation range of the uncertainty

Weight for indicator $\bar{i}$ $y_{r}$

$y_{r}^{\max }, y_{r}^{\min }$

$\boldsymbol{\alpha}$

$\beta$

$\Delta_{i} L(t), \Delta_{i} N L(t)$ $\Delta_{i} P(t)$

$\Delta_{i} P V(T), \Delta_{i} W(t) \quad$ Power ramps in PV and wind

$\Delta p_{\text {avgd+ }+ \text {, }}$

$\Delta p_{\text {avgt }+1-}$

$\Delta p_{\operatorname{maxd}+1-}$

, $\Delta p_{\operatorname{maxt}+p_{-}}$

$\Delta p_{n}$

$\Delta t$

$\mu_{P V}, \mu_{W}$

$\sigma_{d}, \sigma_{t}$

$\sigma_{d+1-}, \sigma_{t+1-}$ wind/PV power output. hours day and at observation time $t$ at observation time $\mathrm{t}$. Time interval. and wind over one year. time $\mathrm{t}$
$\Delta p_{\text {avgd }} \Delta p_{\text {avgt }}$

one of the physical variables of generator $r$

Maximum and minimum specification limi of the physical variable $y$ of generator $r$

The total contribution of wind and solar energy to annual electricity consumption

The share of power output from PV in the

The power ramps in load and net load Power ramp in a given time interval of $i$

The average value of power ramps during the studied day and at observation time $\mathrm{t}$

The average value of upward and downward power ramps during the studied

The maximum value of upward and downward power ramps during the day and

The historical readings for the power change at a selected observation time $t$.

The average value of output power from PV

The standard deviation of power ramps during the day and at a selected observation

The standard deviation of upward and downward power ramps during the day and at a selected observation time $t$

\section{Introduction}

The continuous addition of variable renewable generation (VRG) in the power systems in spite of dispatchable conventional generation that its output can be adjusted according to market conditions has led to increasing the flexibility requirements to deal with power ramps in VRG. the power system flexibility is defined as the use of all the different components of the power system from power sources, infrastructure, operating systems and loads to make the balance between generated and consumed power capacity in all different operational time frames [1]. The concept of flexibility is generalized as "the capability of a power system to adjust the variation and uncertainty through the economic deployment of available resources for a given time interval. From a probabilistic perspective, flexibility indicates the probability that the supply of flexibility is abundant compared to the demand during the period of concern." [2]. At low shares of VRG, the power system operators successfully managed the balance between generation and demand, the required flexibility is provided by reserve generation and generators scheduling as the system demand can be predicted to a large extent and the forecasting error is very small, but at high shares of VRG, a new evaluation of reserve generation is required, as the 
forecasting error is still relatively high and the VRG output power is unclear until it is realized, which require the estimation of the available and required flexibility in the power system. In [3], proposed studying energy-based operating reserves requirements instead of power-based to handle limited energy resources such as storage systems and demand response. Figure 1 shows the effect of wind power generation in net load (net load represent the remaining load that not supplied by VRG) which can cause high power ramps if wind generation decreases at the same time that demand increases, periods of shorter peaks in which conventional power generations operate fewer hours which affect the cost. Higher wind production produces the need for dispatchable generators that can turn their output power down to the low levels throughout the periods of low demand but remain available to rapidly increase it again [4].

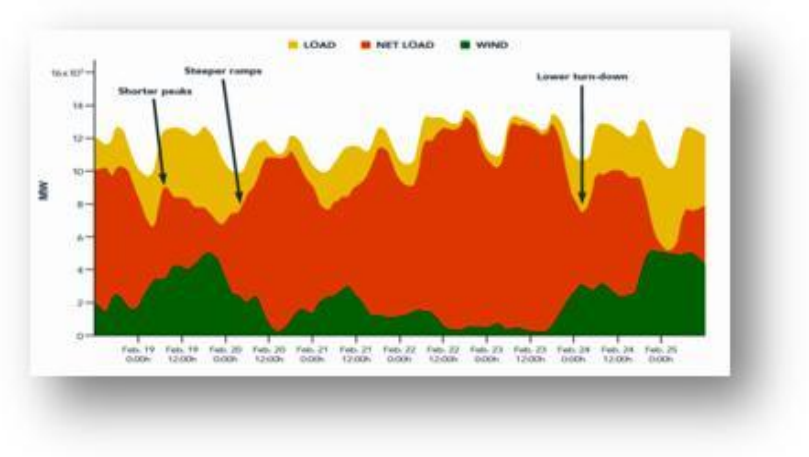

Figure 1. The effect of wind power production in net load

The power system may have sufficient flexibility, but this flexibility is available in certain areas and cannot transfer between different power system areas due to transmission lines constraints. In [5], a new expression called locational flexibility was introduced for describing the power system capability in containing a certain node disturbance, the presented case studies illustrate that flexibility is varied at different grid locations.

\section{Inflexibility features in power systems}

The inflexibility of the power system can be investigated with the following characteristics:

a) Balance violation between demand and generation, leading to deviation of frequency or drop of loads.

b) Curtailments of VRG that mostly happen when there is abundance supply not absorbed by demand or when there are transmission lines limitations.

c) Electricity prices can reflect the inflexibility in the power system which appears in the following forms:

\begin{abstract}
-Negative electricity prices due to many reasons such as conventional generators that could not decrease their output, disability to utilize the surplus generation and constrained capacity of transmission lines to transfer power over more extensive geographic areas. Nevertheless, negative prices sometimes happen without renewable generation in systems but it significantly increases with increasing shares of VRG.

-Instability in electricity prices as prices fluctuate between low and high due to restrictions in transmission lines capacity, inadequate ramping availability, operation of peak load power plants, and limited demand-side response [4].
\end{abstract}

\section{Options for increasing flexibility in power systems}

The transformation of a traditional power system to a VRG based system requires increasing the system flexibility by using the following options[1]:

a. High flexible generators that have fast ramping capabilities.

b. Demand-side Management (DSM).

c. Energy storage systems.

d. Robust communication and control infrastructure.

e. Sufficient transmission lines capacities.

f. Smart grid and internet of energy (IOE).

g. Interconnection and with neighbouring systems.

h. Selecting appropriate operational procedure and advanced forecasting techniques.

i. Transforming excess electricity into thermal energy or to high-value gas products.

j. Coordination between network operators (transmission system operator and distribution system operator)

It should be noted that there is no single flexibility option can meet all the flexibility requirements of the power system efficiently but a combination of flexibility options can do so with high efficiency.

\section{Flexibility and generation adequacy}

For high shares of VRG, the expression of system flexibility is introduced to complement generation adequacy. That can be simply determined, as the load profile used for adequacy calculations is relatively predictable. Generation adequacy is a function of aggregated generators capacity in the system, the forced outage rate of each generator and the yearly peak load hours, while a high degree of uncertainty surrounds system flexibility and its calculation is more complicated and more detailed data are required such as [6]:

a) Availability of each power resource and its ramp rate.

b) Magnitude and duration of net load ramps.

c) Prediction of variations in net load.

d) Interconnection between alternative systems.

e) Existence of storage energy systems. 
f) Demand-side response availability.

g) The arrangements of the market in place.

h) The strategies of reserve provision.

i) Flexibility requirements and resources vary according to the studied time horizon.

\section{Evaluation of flexibility metrics studies}

Flexibility metrics are divided into metrics that measure system flexibility either available or needed and metrics that measure generator flexibility.

\subsection{Generators flexibility metrics}

The power system operational flexibility was quantified and visualized in [7]. Where four parameters were used: power capacity $(\pi)$, ramp-rate $(\rho)$, energy capacity $(\Theta)$ and duration of the ramp $(\delta)$.

Operational flexibility was described as the set-points of all possible operations constrained by the three parameters $\rho \frac{ \pm}{m a x}, \pi_{m a x}^{ \pm}$and $\epsilon_{\max }^{\frac{ \pm}{m}}$. For a power system unit, the three parameters span the flexibility cube, see fig. 2 .

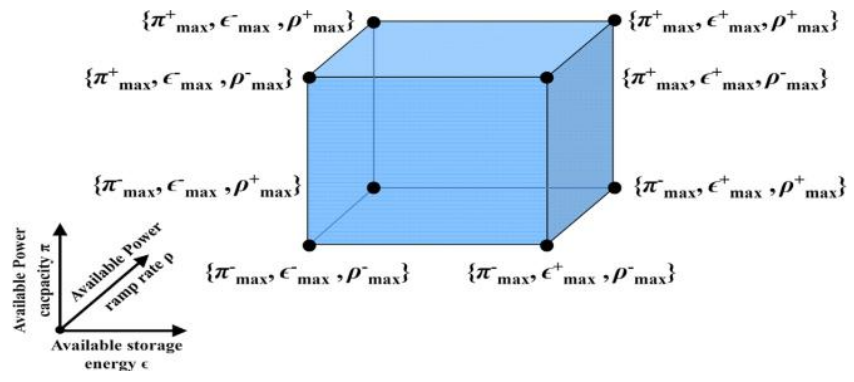

Figure 2. The flexibility cube of a generic power system unit with maximum available operational flexibility

According to the authors, aggregating different units in a power system result in increasing flexibility capability of the sum because their individual parameters of flexibility are added.

In operation, the available flexibility in any power system should be at any case as that needed to mitigate an expected worst-case disturbance; this condition is not only for the average but also for every time-step. For power system accommodation to events that cause a disturbance, the volume of the flexibility available should envelop the volume of the required flexibility. If not, there is at least one of the flexibility parameters axes lacking flexibility and the power system could not completely accommodate the disturbance events. The same concept is illustrated in [8][9], where the authors used dynamic envelopes in characterizing the provision and requirements of flexibility, The authors assert that by enclosing the envelope of flexibility requirement that formed via net load observations by the envelope of aggregated flexibility that shaped by flexibility power resources, sufficient power system flexibility will be achieved. However, the transmission line constraints are not included in the calculations.

The authors in [10] proposed two "offline" metrics, the first called normalized flexibility index that proposed to evaluate the capability of individual generating units and a mixture of generating units in providing the required flexibility. The flexibility index of the individual generator (r) was given by:

$$
\text { Flex }_{r}=\frac{\frac{1}{2}\left[P_{r}^{\max }-P_{r}^{\min }\right]+\frac{1}{2}\left[R R_{r} \times \Delta t\right]}{P_{r}^{\max }}, \forall r \in A
$$

Where $\mathbf{1 / 2} \boldsymbol{R}_{\boldsymbol{r}}$ refers to the average value of the upward and downward ramp rate. The flexibility index of the individual generator $r$ is positive and less than one. The flexibility metric of the entire system $\left(\boldsymbol{F L E X _ { A }}\right)$ was determined by the summation of generators flexibility indices multiplied by a weighting factor that is taken as the capacity contribution of each individual generator.

$$
\text { FLEX }_{A}=\sum_{r \in A}\left[\frac{P_{r}^{\max }}{\sum_{r \in A} P_{r}^{\max }} \times F^{\prime} l e x_{r}\right], \forall r \in A
$$

If the flexibility metric of a certain generator is greater than that of the entire system, this generator in this system is classified as flexible. If-else it is classified as inflexible. The drawbacks of this metric: The classification of thermal generating units as flexible or not is restricted to the studied system and changed from one system to another. The metric is very simple while the operation of the power system is complicated and variable. The metric depends only on two physical characteristics of generator which are the operating range $\left(P_{r}^{\min }, P_{r}^{\max }\right)$ and ramp rate and ignore other physical characteristics such as start-up time, shut-down time, minimum uptime and minimum downtime. In addition, since it is an offline metric, it does not take into consideration the current operating state of the generator, where an online generator with high flexibility could not have the capability of providing more flexibility. Furthermore, the metric focuses only on the flexibility of thermal generation whereas the transmission line constraints, storage and demand-side response and current operational state of generators not included in the flexibility calculations of the entire system.

The metric may be utilized in the early planning stage as it is fast calculated to check the flexibility of a studied system by adding new generators without performing simulation for system operation to evaluate the technical capabilities of system generators to accommodate variable renewable energy sources.

The second offline metric will be discussed in the following sections.

In [11], the authors presented a framework to build up a compound metric for flexibility evaluation within power system conventional generators. In which eight physical characteristics of generating units are used, which are 
maximum output power, minimum stable output level that represent the generator operating range (OR), ramp-up rate (RUR), ramp-down rate (RDR), start-up time (SUT), shutdown time (SDT), minimum uptime (MUT) and minimum downtime (MDT).

The metric calculation contains a sequence of stages (see fig.3), which started by normalization of physical characteristics by using min-max method then weights are assigned to these indicators according to their potential impact in providing flexibility. After that, the indicators are aggregated for each generator to provide the compound flexibility metric.

$$
\begin{gathered}
\text { Flex }_{r}=\sum_{j=1}^{k}\left(I_{j r} \times W_{j}\right) \quad,(j=1, \ldots, k) \\
\sum_{j} W_{j}=1 \\
0 \leq W_{j} \leq 1
\end{gathered}
$$

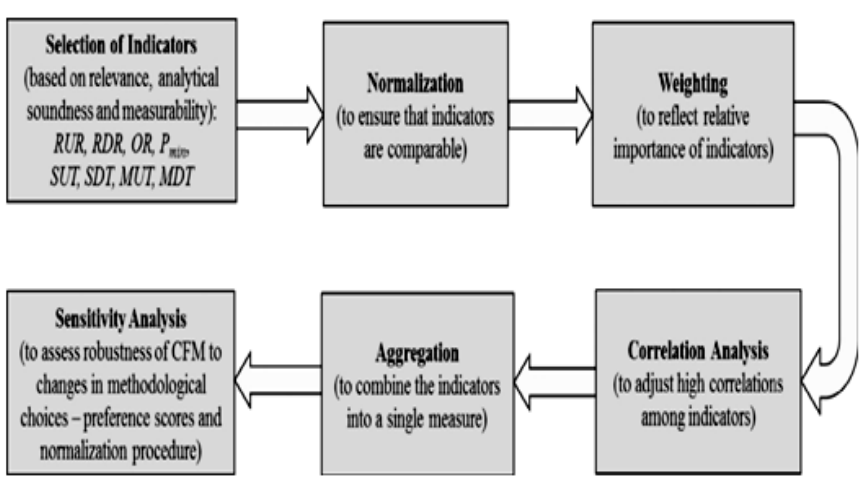

Figure 3. The sequence of steps for building up a compound flexibility metric

The drawback of this metric, it does not take into consideration the current operational state of the generator. Where an online generator with high flexibility could not has the capability of providing more flexibility.

\subsection{Power system flexibility metrics}

In [12], the flexibility of the system is measured over the next five hours. Where flexibility is measured as a large variation range in the uncertainty (v) within which the power system remains feasible under a certain response time and cost threshold divided by the target uncertainty range (V), the system intended to accommodate. The proposed metric takes the transmission line and operational constraints into consideration.

$$
\text { Flex }_{\mathrm{A}}=\frac{w}{V}
$$

The drawback of this method, the degree of flexibility and the frequency of flexibility shortage depend on the risk tolerance of power system operators, as the target uncertainty range or forecast error range is set by the system operators.

The contribution of wind generation in the Irish power system is about $40-50 \%$ of total electricity consumption. The variation in wind production is managed by estimating the flexibility available in the system for the upcoming hour then comparing it to several values of the forecasted net load. From the comparison, the power system operator estimates whether the existing power resources have the capability to balance net load ramps in the presence of an estimated large forecasting error [13][14].

\section{Forecasted net load Ramp + Forecast Error = System Ramping Requirement}

\section{Ramping deficit $=$ Ramping Requirement - Generator Ramping Availability}

The drawback of this method, it does not take into consideration the transmission lines constraints. In addition, the calculation of ramping deficit every hour is a relatively long time interval as the wind production may vary many times during that period.

In [15], the authors used the methodology presented in [16] to represent the possible solutions for all input scenarios in a multi-dimensional space forming the operation region (uncut polyhedron), which is not necessary inside the system capacity available. Restrictions on physical variables corresponding to the available power resources are shaped the outside borders of a region (cube) of constraint specification. The intersection between the operating region and constraint specification region shaped feasibility region (cut polyhedron) which corresponded to the electric potential needs that provided resources should meet. The ratio of feasibility region to the operating region represents the flexibility metric.

$$
\text { Flexibility }=\frac{\text { Volume of cut polyhedron }}{\text { Volume of uncut polyhedron }}
$$

The metric values range from 0 to 1 , fig. 4 shows a graphic illustration for this metric, where $y_{r}$ indicates one of the physical variables that constrained by maximum and minimum specification boundary $\left(y_{r}^{\max } \leq y_{r} \leq y_{r}^{\min }\right)$.

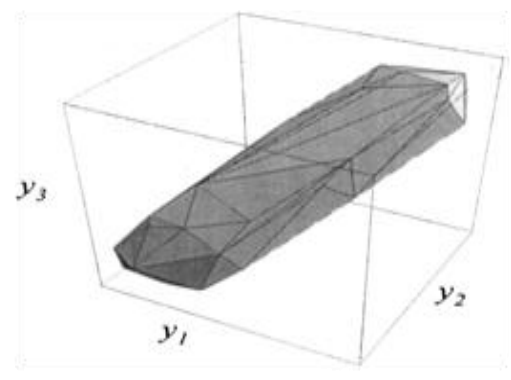

Figure 4. Illustration of the flexibility metric 
The mentioned regions varied according to the studied time, giving a dynamic flexibility metric evaluated over time. The flexibility metric depends on comparing the balance reserves available by the unit commitment and dispatching solutions to a group of VRG output scenarios and system demand. A balancing reserve solution is associated with each scenario provided that it preserves a certain level of reliability but a single solution is kept for committing in advance. Obviously, one scenario's solution doesn't necessarily meet other scenarios' reliability requirements. The sum of the probabilities of the scenarios fulfilled by the selected solution strategy defines the flexibility metric, see fig. 5 .

The drawbacks of this metric: For each period, it presupposed the calculations of a variety of unit commitment scenarios, which increase the computational efforts required. In addition, it does not take into consideration the transmission lines constraints.
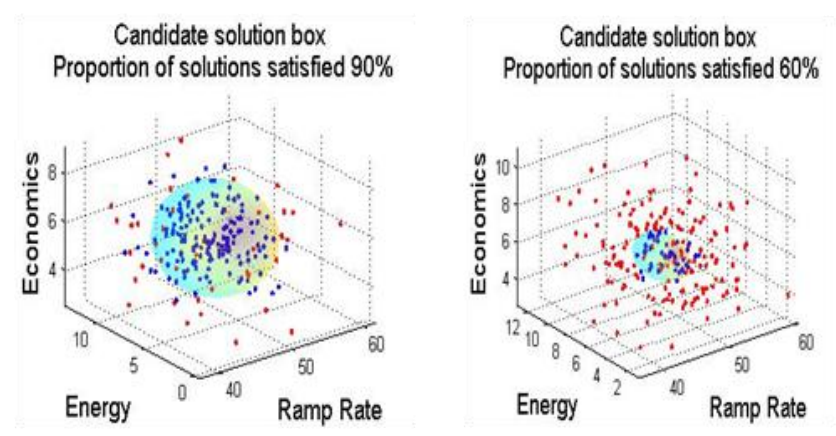

Figure 5. Comparison between high and low flexibility index for a candidate solution strategy

In [17], the authors proposed a metric known as insufficient ramping resource expectation (IRRE) that deduced from generation adequacy indicators for evaluating the flexibility of a power system in the planning stage. In this metric, the ramping in net load time series $\left(\boldsymbol{N L} \boldsymbol{R}_{t_{i} \tilde{i}_{0}+\gamma_{-}}\right)$ is determined in each time horizon then divided to up and down ramps.

$$
\begin{gathered}
N L R_{t, i}=N L_{t}-N L_{t-i} \\
1 \leq t \leq|N L|-i \\
N L R_{t, i+t}=N L R_{t, i} \forall N L R_{t, i}>0 \\
N L R_{t, i-}=N L R_{t, i} \forall N L R_{t, i}<0
\end{gathered}
$$

An offline resource provides flexibility if its start-up time $\left(S_{r}\right)$ is short compared to the studied time horizon with adequate time for reaching the minimum level of stable generation. If a resource achieves this constraint, the available upward flexibility $\left(\right.$ flex $\left._{t, r_{i},+}\right)$ is determined by its ramp-up rate $\left(R R_{r_{2}+}\right)$ by the residual time that results from subtracting its start-up time from the studied time horizon. While ramp-up rate, minimum and maximum generation $\left(\mathcal{P}_{F}^{\max / \min }\right)$ are the necessary constraints for an online resource.

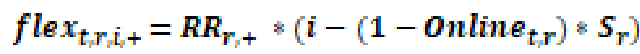

$$
\begin{aligned}
& \boldsymbol{P}_{\mathrm{tr}}+\text { flex }_{\mathrm{tr} i+} \leq \boldsymbol{P}_{\boldsymbol{r}}^{\max }
\end{aligned}
$$

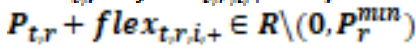

The downward flexibility available for an online resource ( flex $\left._{t_{t} r_{i} i_{2}-}\right)$ is constrained by the ramp-down rate $\left(R R_{r_{i}-}\right)$ and the minimum level of stable generation.

$$
\begin{aligned}
& \text { flex }_{t, r_{i}-}=R R_{r_{i n}-} * i * \text { Online }_{t, r} \\
& 0 \leq P_{t r}-\text { flex }_{\text {tri }}- \\
& P_{t r}-f_{t e x_{i} i-} \in \backslash R\left(0, P_{r}^{m u n}\right)
\end{aligned}
$$

For each resource, once both upward and downward flexibility are determined for wholly studied time horizons, the time series of system flexibility $\left(\right.$ flex $\left._{t S Y S T E M_{2} i_{2}+f_{-}}\right)$is calculated as follows:

$$
\text { flex }_{t, \text { SYSTEM }, i,+/-}=\sum_{\forall r} \text { flex }_{t, r, i,+/-}
$$

After that the available flexibility distribution $\left(A F D_{i_{i}+\gamma-}(X)\right)$ is deduced from flex $_{t}$ SYSTEM $_{i_{2}+\gamma-}$, which indicates the likelihood that power of X MW or lower will be available from a flexible power resource during the time horizon $i$, then the insufficient ramping resource probability $(I R R P)$ for each observation time is calculated from $A F D_{i_{i}+\gamma-}(X)$, which represent the cumulative probability that a power system failed to meet the required net load ramp at that observation time. In order to eliminate cases at which just enough flexibility is available in $A F D_{i+\gamma-}(X)$, the magnitude of net load ramp is reduced by $1 \mathrm{MW}$. Hence, IRRPis given by:

$$
I R R P_{t_{i} i_{j} j_{-}}=A F D_{i_{j}+j^{-}}\left(N L R_{\left.t_{i}+j_{j}-1\right)}\right.
$$

The summation of $I R R P_{t_{i} i_{3}+f}$-for each ramp direction over the whole time series $\left(T_{+/}\right)$gives the insufficient ramping resource expectation $\left(I R R E_{\tilde{i}_{1}+/-}\right)$ as follows:

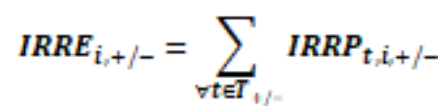

The drawback of this method, it does not take into consideration the transmission lines constraints as sufficient ramping capabilities may be available in the system but cannot transfer between different areas.

In [6], the authors studied system flexibility at planning stage hence some assumptions were supposed, such as the generator's operation is dependent on the merit order dispatch, by which the demand is supplied by dispatching the generating units according to the incremental marginal 
cost of energy at the maximum output from each generator. The proposed methodology determines the periods of flexibility deficit which requires the output power time series from each flexible resource in the system such as generators, storage systems, demand-side response (DSR), and from the interconnection with adjacent areas. The periods of flexibility deficit are calculated as follows:

(i) The average cost (AC) for each generating or storage unit at maximum power output is determined then the power resources are arranged in ascending order in accordance to the increase in marginal costs to form the merit order supply function.

\section{$A C=\frac{\text { Noloadcost (NRL) }+ \text { CAPACITYMarginalCost }[\text { MC) }}{\text { CAPACIYY }}$}

(ii) The net load time series is calculated by subtracting the output of VRG from system demand at each observation time then net load ramps (NLR) are determined.

$$
\begin{gathered}
\mathrm{NL}_{\mathrm{t}}=\text { system demand }_{\mathrm{t}}-\sum V R G_{\mathrm{t}} \\
N L R_{t, i}=N L R_{t+i}-N L R_{t} \\
1 \leq t \leq|N L|
\end{gathered}
$$

(iii) The net load duration curve (NLDC) is formed by arranging the net load in descending order. For system reserve provision, the required reserve can be added to NLDC. After that, the net load is supplied by adding resources One after the other starting with the least cost according to the merit order and by taking into consideration minimum stable generation level for each generator (MSG).

(iv) The available flexibility from an online power resource $\left(\mathrm{F}_{\mathrm{t}, \mathrm{i}, \mathrm{F}}^{\mathrm{Onlime}}\right)$ is determined by calculating the maximum increase in its power at the studied time horizon which constrained by its rated power and the initial output power at each observation time:

$F_{t, i, r}^{\text {Online }}=$ Online $_{t, r} \times \min \left(R R_{r} \times i\right.$, Rated capacity $\left.r-P_{t, r}\right)$

(v) The flexibility available from an offline resource if it can be synchronized and start production within the selected time horizon is given by:

$\mathbf{F}_{\mathrm{t}, \mathrm{i}, \mathrm{r}}^{\text {Offline }}=\min \left(\mathbf{R R}_{\mathrm{r}} \times\left(\mathbf{i}-S_{r}\right)\right.$, Rated capacity $\left.r\right) \times\left(1-\right.$ Online $_{\mathrm{t}, \mathrm{r}}$

$\forall \mathrm{i} \geq S_{r}$

(vi) The time series of the flexibility available for a power system is calculated by adding both offline and online available flexibility for every power resource.

$$
F_{\text {ti }}^{\text {ayailable }}=\sum_{r=1}^{K} F_{\text {tir }}^{\text {Online }}+\sum_{r=1}^{K} F_{t_{i} i r}^{\text {Offline }}
$$

The drawbacks of dispatching generators by merit order method are the exaggeration the system's flexibility, as the results, in reality, are sub-optimal because the merit order commitment is non-chronological and the power system costs are minimized for each time interval, while unit commitment solutions take many other factors such as demand forecasting of next periods, forecast errors, start-up time and cost for each power resource. Consequently, dispatching fast-starting resources that are more expensive out-of-merit is possible to avoid the start-up of huge power resource that is only required for a small period of time. Therefore the flexibility available is less than that of merit order method. Moreover, the transmission network constraints are not included. In addition, although net load ramps may be up or down, the upward flexibility was examined only.

As a result, an improvement to the previous method for more realistic results is done by mixing between merit order and economic dispatch methods, in which all power resources are dispatched by merit order in condition that their maximum power output is less than net load level and the remaining resources are dispatched for supplying the residual of net load according to the economic dispatch method to reduce the total cost. A power system is considered has a shortage of flexibility or net ramping resource deficit $\left(P F D_{t, i}\right)$ when $N L R_{t, i}$ is greater than the flexibility available $\left(F_{t_{e} i}^{\text {available }}\right)$.

$$
\boldsymbol{P F D}_{t, i}=N L R_{t, i}-F_{t i}^{a \text { avaliate }}
$$

$P F D_{i_{3}+\gamma_{-}}$is equivalent to the IRRE outlined in [17], as both metrics measure the system flexibility, but differ in the assumptions relating to the risk level and $P F D_{t, i}$ is deterministic but $I R R E_{\tilde{i}+/}$ - is a probabilistic metric.

The drawback of this method, the transmission line thermal limits and security constraints are not included in the calculations.

In [18], the authors proposed a simple metric based on dividing the net load ramping by the available power system flexibility, when the metric less than 1 , there is sufficient flexibility in the system. While load shedding is required if the metric is more than 1 , as follows:

$$
\text { flex }_{t \text { SYSTEM } i=}=N L R_{t, i} / F_{t i}^{a y n i l a b l e}
$$

The drawback of this method, it does not take into consideration the transmission lines constraints as sufficient ramping capabilities may be available in the system but cannot transfer between different areas.

In [19], the authors presented a metric for power system planners to calculate both upward and downward flexibility and to demonstrate the effect of transmission line networks on the flexibility by calculating maximum realizable flexibility. The maximum demand that can be added to a power system and balanced by the system power resources without breaking either the generating units or the network restrictions was defined as the maximum flexibility that can be realized for that period of time $(R F)$.In this method 
upward and downward flexibility are calculated as in [17]. If the power system network is not congested, all flexibility available from every power source that scheduled for operating will be realized. But in fact, the total realized flexibility may be lower than that typically available because the flexibility estimation methods depended only on the resource schedules, which led to an overestimation of system flexibility. For calculating realizable flexibility, specialized computer programs should be used such as the one used in [20], by which an optimization process can be performed to calculate the maximum allowable power system flexibility under a variety of conditions for all time periods. Through the optimization process the net demand, and consequently the output power of flexible power resources are increased until reaching either network or flexibility limitations. In that case, the maximum upward flexibility that can be realized is determined. After that, the path of the optimization process is altered to calculate the flexibility which can be realized downward. The realized flexibility is estimated by considering three varied parameters, both of them are time-dependent which are the time period and the selected time horizon $(t, i)$ and the other varied parameter is the output scenario of VRG $(s)$. For every scenario, a different flexibility amount is deployed according to ramps in VRG until the line flow boundaries were reached. Hence, different values of flexibility are estimated according to the studied scenario and the expected values at every time period are taken to represent the flexibility of the system.

$$
\begin{aligned}
& \text { NetFlexibility } \text { tis }+_{+}=R F_{\text {tis }}-\left(\text { Contingency }_{t_{+}+}+N L R_{t i}\right)^{-} \\
& \text {NetFlexibility } \text { tis }_{-}=N L R_{t i}-\text { Contingency }_{t_{-}-}-R F_{t i s} \\
& P F D_{s i+f-}=\text { NetFlexibilitytis }+1-1<0 . \\
& P F D_{i_{i}+j_{-}}=\operatorname{Mean}\left(\mathrm{PFD}_{\boldsymbol{s i}_{i}+j_{-}}\right)
\end{aligned}
$$

The metric of flexibility deficit periods $\left(\boldsymbol{P F D _ { s _ { i } i _ { j } } )}\right.$ ) counts the number of time periods at which the summation of net load ramping $\left(\boldsymbol{N} L \boldsymbol{R}_{t i \boldsymbol{i}}\right)$ and contingency reserve (Contingency $y_{t}$ ) requirements exceed the realized flexibility $\left(\boldsymbol{R} \boldsymbol{F}_{t i s}\right)$ for each ramp direction. The index of $\boldsymbol{P} F \boldsymbol{D}_{s i+t-}$ is calculated for each scenario and after that, the $\boldsymbol{P F} \boldsymbol{D}_{i_{i}+\gamma-}$ for that time horizon and direction were taken as the average of all scenarios values.

The drawbacks of this index, the transmission line thermal limits and security constraints are not included in calculations. In addition, for each scenario of VRG production, a different flexibility amount is deployed according to ramps in VRG and consequently a different number of periods of flexibility deficit which increase the computational efforts required.

In [21], wind and solar power that integrated with different combined penetration levels in Europe were discussed. The analysis concluded that the important element was the power ramps $\left(\Delta_{i} \boldsymbol{P}(\boldsymbol{t})\right)$ which represented by the variation of power in a studied time interval of (i) hours as follows:

$$
\begin{gathered}
\Delta_{i} P(t)=p(t)-p(t-i) \\
t=\{i+1, \ldots ., 8760\}
\end{gathered}
$$

The net load ramp rates are determined for every country as a function of load, wind and PV ramp rates as follows:

$$
\begin{aligned}
& \Delta_{i} N L(t)=\Delta_{i} L(t)-\frac{\alpha \beta D}{8760 \mu_{p v}} \Delta_{i} P V(t) \\
& -\frac{\alpha(1-\beta) D}{8760 \mu_{W}} \Delta_{i} W(T)
\end{aligned}
$$

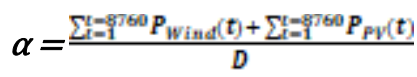

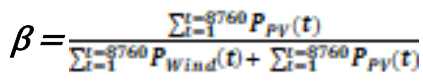

For performing a comparison between countries, all ramps occurred in every country were determined as a percentage from the peak load. The system requirements of flexibility were evaluated depending on the following factors:

(i) The VRG share and the share of wind and PV in the wind/PV generation as choosey variables selected by decision-makers.

(ii) The characteristics of power ramps in load, PV and wind which affected by the geographic location, the positioning of the generator and the size of the system.

(iii) The correlation between ramps in VRG and load and also between wind and PV ramps.

The drawback of this method, it does not take into consideration the transmission lines constraints. In addition, the calculations of power ramps in a studied time interval of (i) hours are relatively a long time interval as the wind and PV production may vary many times during this period.

In [22], a flexibility chart was developed to present a simple non-technical means to quickly identify the potential of the system's flexible power resources. In which the installed capacity percentage of five flexible power resources including combined cycle gas turbine, hydro, pumped hydro, combined heat and power (CHP) and interconnection relative to the peak demand were indicated in the chart. However, the chart was just for indication and did not include the calculation of the overall flexibility of the power system.

In [23], a structure for flexibility planning model was proposed. The flexibility needs are determined by running an optimization algorithm over the whole year with a onehour resolution, the optimization results determine the number of generating units of each type (base, intermediate and peaking generating units) that needed to minimize the total cost.

The drawback of this method, the optimal amount of flexibility is investigated depending on the average representative weeks which insufficient to provide inter- 
hour variation of the renewable generation, where the load profile of the whole year is represented by four weeks, each week reflects the average load profile of the season; these weeks represent the 672 hours of the optimization horizon. Furthermore, the transmission line limitations are not included in the calculations.

In [24], a flexibility ramping product model is proposed that based on net load forecasts, in which joint probability distribution of load, wind and solar forecasting errors are used to cover all extreme forecasting errors. In [25], the design of flexibility ramping product is proposed by modelling uncertainty through simulation-based optimization for selecting net load standard deviations.

The drawback of these methods, the flexibility ramping product is only focused on conventional generators rather than other sources such as storage systems and demand-side management. Furthermore, the transmission line congestion is not taken into consideration to ensure the delivery of flexibility offered by different generating units located at different locations in the power system. The case study used in [25] is very simple and for proving the method, a practical case study is required.

In [26][27], the authors estimated the flexibility range for the next hours at each primary substation node, as the feasible flexibility resources that are available in the distribution grid which originated from actions of flexibility (demand response, flexible distributed generation, or control of reactive power from the distribution system operator assets) are informed to the transmission system operator. The costs of activating these flexibility resources are also considered to carry out a cost-benefit assessment of the available actions.

The drawback in [26], the random sampling by the Monte Carlo simulation is not the best solution as it is difficult to capture the primary substation's extreme flexibility points, even if the number of samples is increased. Hence, to solve this problem, an optimization model should be developed as well as to reduce the computational effort needed to identify the area of flexibility. In addition, other types of flexibilities, such as flexible AC transmission systems (FACTS) and distributed storage, as well as asymmetric flexibility costs should also be included in the methodology. While an optimization model was used in [27] to explore the limits of active and reactive power flow at the boundary nodes that define the flexibility area. However, further investigations should be carried out to study the effect of assets of a discrete control nature (e.g., on-load tap changers, capacitor banks) on the occurrence of disjoint flexibility areas.

\subsection{Power system flexibility evaluation tools}

The International Energy Agency (IEA) designed a flexibility assessment tool (FAST) which utilized as a measure of flexibility requirements in various power system areas with different VRG penetration levels [28]. In 2014, IEA presented FAST2 which is a modified version of FAST with many flexibility assessment time scales [29]. More data is needed by FAST2, such as the flexibility features of conventional units, the information about DSM and interconnection. FAST2 determines the maximum alteration in the balance between generation and demand at a given instant that the power system is able to meet. In addition, it computes the VRG integration level at which more flexibility is needed.

In [30], The International Renewable Energy Agency (IRENA) has designed an open-source tool to evaluate power system flexibility for members (Colombia, Uruguay, Panama, and Thailand) and to identify least-cost solutions to efficiently increase VRG's share of their energy mix. The FlexTool is data-driven, which means that the model structure is relatively general, and input data plays a major role in specifying what the model does. The inputs required include demand, generation mix, VRG time series, interconnections and fuel costs.

The Electric Power Research Institute (EPRI) made a framework consists of four flexibility evaluation levels at various stages of power systems long-term planning process [31][32]. InFLEXion is a multi-level flexibility evaluation tool that includes four flexibility metrics which are IRRE, PFD, expected unserved ramping (EUR) and well-being assessment. The expected unserved ramping metric evaluates the total flexibility shortage magnitude rather than duration; it determines the aggregate deficits of power ramping over a certain time horizon. Well-being analysis used the deterministic and probabilistic indicators to identify the state of the power system from the following states: at risk, marginal or healthy [33]. Similarly, InFLEXion tool evaluates the flexibility shortages magnitude and frequency over a specified time period using PFD and EUR to decide whether the state of the power system is safe, warning, or dangerous [32].

In [34], the authors noted that the earlier studies concentrated mostly on the operating problems and did not address the economic considerations of adding flexibility resources to the power system. As a result, a simulation model was developed for stochastic production, known as REFLEX (Renewable Energy Flexibility), in which a variety of power system reliability and flexibility metrics are used in characterising the flexibility expected and shortages of adequacy in the system, it then evaluates the optimal investment in flexible capacity by comparing the additional costs of flexible resources with the improvement obtained as a result of avoided violations of flexibility. This assisted power systems planners in the determination of least-cost capacity strategy to face challenges introduced by the integration of VRG in various timescales.

\subsection{Flexibility metrics derived from generation adequacy metrics}

Some of the power system flexibility metrics are derived from the generation adequacy metrics. The authors in [35] used the effective load carrying capability (ELCC) method [36][37], which measures the added load that the power system is able to supply with a particular generator without 
any change in reliability to suggest the effective ramping capability metric (ERC). ERC metric is used in estimating the contribution of a new generating unit to overall power system capacity; in addition to the unit's contribution to the ramping capacity of the overall power system. The calculation of ERC is the same as that of ELCC, except that, rather than using the rated output power of the unit, the maximum ramp of the unit is applied in a certain direction and time period.

The drawback of this method is in calculating the ramp availability rate of conventional generation plant based on a simple merit order. Furthermore, it does not take into consideration the transmission lines constraints as sufficient ramping capabilities may be available in the system but cannot transfer between different areas.

In [38], the authors presented a framework for characterizing the available flexibility in the multi-area systems, The framework is focusing on the available reserves and the tie-lines flow, which is similar to the available transfer capacity (ATC) metric, but (N-1) security criterion is taken into consideration. The proposed approach discussed the inclusion of flexible resources in adjacent areas to handle the occurred contingencies in a certain area. Hence, the term "exportable flexibility" was introduced to measure the flexibility that one area can introduce to its neighbours. This enables the power system to handle larger variations and permits more integration of VRG.

The methodology drawback is in the available flexibility calculation, which is based on the assumption that the demand is covered and the operational restrictions are achieved. Also for validating the methodology, more examples that are focusing on more than two areas are required.

In [39], the lack of ramp probability (LORP) was proposed, which is similar to the loss of load probability (LOLP) that is utilized for measuring capacity adequacy. LORP measures the capability of the system for meeting net load changes in real-time. LORP is relied on the possibility of meeting the expected net load in the next period from the current generator schedules, considering the inter-temporal schedules increments of both imports and exports interconnection, in order to add the positive import and negative export increases as ramp-up capacity as follows:

$$
\begin{aligned}
& \begin{array}{c}
\operatorname{LORP}_{S}^{+i}[i t]=\operatorname{Pr}\left(\sum _ { r \in A } \left\{P_{r}^{g}[t]+\operatorname{mĩn}\left(i \times R_{r^{v}} P_{r}^{\max }-P_{\eta}\right.\right.\right. \\
\left.<N L_{s}[t+i]\right)
\end{array}
\end{aligned}
$$

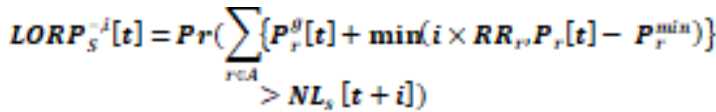

$$
\begin{aligned}
& R C_{\bar{u}}[t]=\text { Zonal Generator RampPower }[t]+(\operatorname{Import}[t+ \\
& \text { - Import }[t])-(\text { Export }[t+1]-\operatorname{Export}[t+1])
\end{aligned}
$$

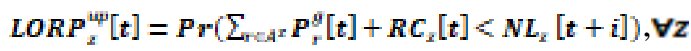

The proposed metric computed the available flexibility in two-steps: (i) The network is reduced first by considering each zone as a single bus with net injection; in which all generators and loads are connected to this bus, the transmission lines flow limits are ignored, and the tielines between each pair of zones are aggregated to an equivalent single tie line. Then in the first step, generation is dispatched to meet the net load of current time period plus the worst case of the following ones, taking into consideration uncertainty and ramps limits.

(ii) In the second step, the flow limits of tie-lines are partially relaxed based on acceptable selected shortterm flow violation for tie-line, which is selected by the system operator, then a deterministic optimization economic dispatch is executed for all the system with keeping the ramping capability of the zones at the same values determined in the first step.

For the current time interval and future time intervals, the generator dispatch solutions are obtained each time the twostep economic dispatch is solved. The current interval solution is implemented whereas the future interval solutions are considered as recommended. After that, the process is repeated by moving forward in time and taking the updated forecast information of both load and renewable generation.

The drawback of this method is in the first step of the dispatch model, as the constraints of intra-zonal flow are not considered, so not all the realizations of the net load uncertainty are taken into account by the dispatch solution. Therefore, shortage events are reduced but not eliminated completely.

In [10], the second offline metric is known as loss of wind estimation (LOWE), which is similar to LOLP. LOWE was proposed to evaluate the power system flexibility by calculating the probability of having wind curtailment in the system during a year. In this metric, statistical analysis is performed to calculate the probability of net load to violate system technical thresholds. The technical thresholds that are taken into consideration are the minimum load level and both upward and downward ramp capabilities.

The drawbacks of this metric, it takes only the balancing issues in wind curtailment and not takes into consideration the transmission lines constraints. In addition, since it is an offline metric, the current operating state of generators is not included in the calculation. Furthermore, it assumes that wind curtailment occurs due to three independent events, when net load decreases below the minimum load level of the system or the violation of either upward or downward capabilities of generators, while the net load can fall below the system's minimum load point, and the generators do not have adequate ramp-down capabilities at the same time.

In [40], The authors proposed new metrics to evaluate power system flexibility by involving electric vehicles into the flexible ramp market. The impact on power system reliability and flexibility were evaluated for both the electric vehicles direct participation and by cooperation with conventional generators. The drawback of these metrics, the calculations do not take into account the transmission lines constraints. 
In [2], four metrics were proposed by the authors which are loss of flexibility probability (LOFP), loss of flexibility duration (LOFD), loss of flexibility expectation (LOFE) and flexibility demand shortage (FDS). The LOFP is similar to LORP and IRRE but LOFP is independent with time, while LORP and IRRE are time-varying. So do as LOFD and LOFE compared to PFD and the expected energy not served (ENS). The metrics have the following characteristics:

- In case of upward flexibility, the relationship between the index and load loss is linear and so as with VRG curtailment in case of downward flexibility.

- The proposed flexibility metrics can be determined, once the acceptable curtailment limit was determined,

- The metrics can be used with diverse options of flexibility such as units of conventional generating and energy storage.

The drawback of these metrics, the calculations do not take into account the transmission lines constraints.

\section{Flexibility and analysis of power ramps in VRG}

The flexibility metrics studies that are illustrated in the previous sections outlined that the important factor in power system flexibility is the power ramps in VRG. While an improvement in wind power forecasting has been achieved, the percentage forecasting error is still relatively high. Therefore, as the VRG shares increases in the system, as the forecasting error impacts the balance between generation and consumption. Consequently, the power system operators should have more information about the behaviour of VRG power ramps in the system, which includes the scale of power ramps as well as the expected times at which high power ramps are most likely to occur, to balance these ramp events in order to achieve flexibility and reliability in the power system. In the next sections, an analysis method for historical VRG data will be presented in order to obtain information about the behaviour of VRG power ramps. The analysis method will be demonstrated by analysing the historical output power of aggregated Belgian wind farms every 15 minutes for the years 2017, 2018.

\subsection{Analysis procedures}

The analysis of historical VRG output in the power-time curve will take two directions, which are vertical and horizontal as follows:

Vertical analysis direction

In which the power ramping behaviour at each observation time $(\mathrm{t})$ in the power-time curve is studied separately in detail. The historical readings are used to calculate the power ramps occurred at a certain observation time within a selected time interval $(\Delta \mathrm{t})$, where $\Delta \mathrm{t}$ is selected by the power system operator according to the studied operational stage, then moving to the next one until finishing all observation points in the power signal time series as follows:

$$
\Delta p_{n}=P\left(t_{n}+\Delta t\right)-P\left(t_{n}\right), \quad n=(1, . ., N)
$$

In the previous equation, both $\mathrm{t}$ and $\boldsymbol{\Delta} \mathbf{t}$ are fixed and $\mathrm{n}$ is changed until finishing all the studied historical readings then moving to the next observation time $(\mathbf{t}+\Delta \mathbf{t})$. If $\Delta p_{n}$ is positive, it refers to power ramp-up $\left(\Delta p_{n_{4}}\right)$. Conversely, if $\Delta p_{n}$ is negative, it refers to power ramp down $\left(\Delta p_{n_{-}}\right)$.

\section{If,}

and if,

$$
\Delta p_{n}>0 \rightarrow \Delta p_{n_{+}}(\text {Ramp-up) }
$$

$$
\Delta p_{n}<0 \rightarrow \Delta p_{n-} \text { (Ramp-down) }
$$

The average value ( $\left.\Delta \mathrm{p}_{\text {avgt }}\right)$ and the standard deviation $\left(\boldsymbol{\sigma}_{t}\right)$ of power ramps at the studied observation time $(\mathrm{t})$ is given by:

$$
\begin{gathered}
\Delta p_{\text {avgt }}=\frac{1}{N} \sum_{n=1}^{N} \Delta p_{n} \\
\sigma_{t}=\sqrt{\frac{1}{N-1} \sum_{n=1}^{N}\left(\Delta p_{n}-\Delta p_{\text {avgt }}\right)^{2}}
\end{gathered}
$$

If $\Delta$ pangt $_{\text {in }}$ is positive, it means that the mostly occurrence ramp type at observation time $t$ is ramp up. Conversely, if $\Delta \mathrm{p}_{\text {avgt }}$ is negative, it means that the ramp down type is the mostly occurrence ramp in that observation time, which give the power system operator the information about the most frequent ramp type at each observation time $t$.

A low standard deviation value indicates that the historical power ramp readings at this observation time are concentrated around the average which means that the average value of power ramps can express perfectly the ramps in the system at this observation time, while a high value indicates that power ramps are spread out over a wider range and the average value does not express perfectly the ramps in that observation time.

The average value and the standard deviation of upward and downward power ramps at the studied observation time (t) are given by:

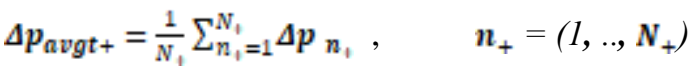

$$
\begin{aligned}
& \Delta p_{\text {avgt- }}=\frac{\mathbb{1}}{N_{\varpi}} \sum_{n_{-}=\mathbb{I}}^{N_{\varpi}} \Delta p_{n_{-}}, \quad n_{-}=\left(1, . ., N_{-}\right)
\end{aligned}
$$

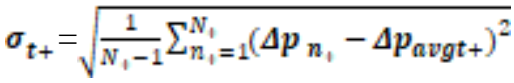

$$
\begin{aligned}
& \sigma_{t-}=\sqrt{\frac{1}{N_{-}-1} \sum_{n_{-}=1}^{N_{-}}\left(\Delta p_{n_{-}}-\Delta p_{\text {avgt }-}\right)^{2}}
\end{aligned}
$$


The maximum historical power ramps and the ramping range at each observation time during the studied time interval can be obtained from "(28)" as follows:

$$
\begin{aligned}
\Delta p_{\operatorname{maxt+}} & =\max \Delta \mathrm{p}_{n} \\
\Delta p_{\operatorname{maxt}-} & =\min \Delta \mathrm{p}_{n} \\
\text { Ramping range } & =\Delta p_{\operatorname{maxt+}}-\Delta p_{\operatorname{maxt}-}
\end{aligned}
$$

After finishing the above calculations at observation time $t$, the next observation time $(t+\Delta t)$ in the power signal time series is taken as the observation time $t$ and the calculations are repeated until reaching $\mathrm{t}=24 \mathrm{~h}$.

\section{Horizontal analysis direction}

In which the daily historical readings of power ramps are studied to get information about the power ramping behaviour in certain weeks, months, seasons or years during the studied time interval $\Delta \mathbf{t}$, the power ramps time series can be obtained by fixing $\Delta \mathrm{t}$ and changing $\mathrm{t}$ as follows:

$$
\begin{gathered}
\Delta p_{t}=P(t+\Delta t)-P(t), \mathrm{t}=(1, \ldots, h) \\
\Delta p_{t}>0 \rightarrow \Delta p_{n+} \text { Ramp-up } \\
\Delta p_{t}<0 \rightarrow \Delta p_{n-} \text { Ramp-down }
\end{gathered}
$$

The average value of power ramps $\left(\Delta \mathrm{p}_{\text {avgd }}\right)$ in the selected time interval $\boldsymbol{\Delta t}$ during the studied day and the standard deviation $\left(\sigma_{d}\right)$ is given by "(38)", “(39)":

$$
\begin{aligned}
& \Delta p_{\text {augd }}=\frac{1}{h} \sum_{t=1}^{i n} \Delta p_{t} \\
& \sigma_{d}=\sqrt{\frac{1}{h-1} \sum_{t=1}^{h}\left(\Delta p_{t}-\Delta p_{\text {avgd }}\right)^{2}}
\end{aligned}
$$

The average value and the standard deviation of upward and downward power ramps during the studied day is given by:

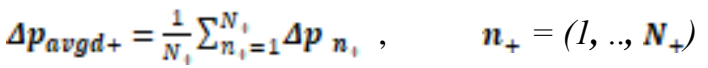

$$
\begin{aligned}
& \Delta p_{\text {avgd }-}=\frac{1}{N_{-}} \sum_{n_{-}=1}^{N_{\infty}} \Delta p_{n_{-}}, \quad n_{-}=\left(1, . ., N_{-}\right) \\
& \sigma_{d+}=\sqrt{\frac{1}{N_{\phi}-1} \sum_{n_{\ell}=1}^{N_{Q_{b}}}\left(\Delta p_{n_{\phi}}-\Delta p_{\text {avgd+ }}\right)^{2}} \\
& \sigma_{d-}=\sqrt{\frac{1}{N_{-}-1} \sum_{n_{-}=1}^{N_{-}}\left(\Delta p_{n_{-}}-\Delta p_{\text {avgd- }}\right)^{2}}
\end{aligned}
$$

The maximum power ramps and the ramping range in a studied day through the studied time interval is given by "(44, 45)":

$$
\begin{gathered}
\Delta p_{\operatorname{maxd}+}=\max \Delta p_{\mathrm{t}} \\
\Delta p_{\operatorname{maxd}-}=\min \Delta p_{\mathrm{t}}, \mathrm{t}=(1, \ldots, h)
\end{gathered}
$$

$$
\text { Ramping range } e_{d}=\Delta p_{\operatorname{maxd}+}-\Delta p_{\operatorname{maxd}}
$$

The historical ramping features in a weak, month, season, and a year ago can be obtained as in "(46)":

$$
\begin{gathered}
\text { his } \Delta p_{\text {avgd }}=\frac{1}{d_{n}} \sum_{d_{1}}^{d_{n}} \Delta p_{\text {avgd }} \\
\text { his } \sigma_{d}=||_{d_{1}}^{d_{n}} \sqrt{\frac{1}{h-1} \sum_{t=1}^{h}\left(\Delta p_{t}-h i s \Delta p_{a v g d}\right)^{2}} \\
\text { his } \Delta p_{\operatorname{maxd}+}=\left.\max \right|_{d_{1}} ^{d_{n}} \Delta p_{\operatorname{maxd}}+ \\
\text { his } \Delta p_{\operatorname{maxd}-}=\left.\min \right|_{d_{1}} ^{d_{n}} \Delta p_{\operatorname{maxd}-}
\end{gathered}
$$

i.e. for a week, month and year, $d_{n}=7,30$ and 365 respectively.

\subsection{Ramp characteristic indicators (RCI)}

The power systems increase the installed capacity of VRG continuously. Consequently, the power ramping behaviour of VRG will also change. The power systems will need to investigate the effect of increasing shares of VRG on the power ramping behaviour. The ramp characteristic indicators are proposed for comparing the ramping behaviour of VRG in different years. These indicators can be utilized also for comparing the ramping behaviour between power systems in different countries, in which the elements of ramping behaviour that calculated in the previous section are divided by the average installed VRG capacity throughout the year as follows:

$$
\begin{aligned}
R C I_{\max +f_{-}} & =\frac{\Delta p_{\max +f_{-}}}{\text {average installed VRG capacity }} \\
R C I_{\text {avg }+f_{-}}= & \frac{\Delta p_{\text {avg }+f-}}{\text { average installed VRG capacity }}
\end{aligned}
$$

$$
\begin{gathered}
R C I_{\text {ramping range }}=\frac{\text { Ramping Range }}{\text { average installed VRG capacity }} \\
R C I_{\sigma_{a, j}}=\frac{\sigma_{+f-}}{\text { average installed VRG capacity }}
\end{gathered}
$$

Frequency of upward and downward power ramps In statics, the frequency of an event refers to the number of occurrences of that event. The relative frequency is calculated by dividing each event frequency by the total number of data points. The sum of events relative frequencies should be equal to 1 . The power system operator can get information about the probability of occurrence of a certain ramp direction as follows:

$$
P\left(N_{+/-}\right)=\frac{N_{+f-}}{N}
$$




\section{Case study}

Belgium is one of Europe's most interconnected countries, as Belgium is capable of importing and exporting over $40 \%$ of its peak demand. In Belgium, coal-fired units were gradually replaced by gas-fired units until the last coal-fired plant was closed in 2016. Furthermore, nuclear power, which accounts for $50 \%$ of the total electricity generated, is also planned to be phased out over the next five years. Accordingly, Belgium's share of renewable generation is expected to double in 2030 compared to 2017 that represented around $17 \%$ of electricity consumption. The output power of Belgium's aggregated wind farms (onshore and offshore wind farms) is analyzed in the last two years 2017 and 2018 using the analysis procedures explained above. The aggregated wind power output in Belgium was recorded every 15 minutes, including the aggregated wind power in Belgium recorded by month, day, hour and minute [41]. The maximum installed wind power capacity in 2017 is $2621.924 \mathrm{MW}$ and the average installed wind capacity throughout the year is $2439.074 \mathrm{MW}$. Whereas in 2018, the maximum installed wind power capacity is $3157.185 \mathrm{MW}$ and the average installed wind capacity throughout the year is $2922.08 \mathrm{MW}$. The variations in wind power are calculated in that time interval ( $\Delta \mathbf{t}=15 \mathrm{~min}$.). The power ramp is considered as a ramp event if it exceeds $5 \%$ of the rated power $\left(p_{R}\right)$.

\subsection{Vertical analysis results and discussion}

A comparison between the total numbers of upward power ramps in 15 min interval at each observation time $t$ for years 2017 and 2018is showed in fig.6, in which the numbers of upward power ramps are nearly equal for all the studied observation times. The numbers of upward power ramps increase in the period from $11 \mathrm{AM}$ to $11 \mathrm{PM}$.

In fig.7, a comparison between the total numbers of downward power ramps in $15 \mathrm{~min}$ interval at each observation time $\mathrm{t}$ for the years 2017 and 2018is showed. The two curves nearly have the same values in most observation times. The numbers of downward power ramps decrease in the period from $11 \mathrm{AM}$ to $11 \mathrm{PM}$. Therefore the numbers of upward power ramps are more than the numbers of downward power ramps in the period from 11 AM to 11 $\mathrm{PM}$, whereas, in the period from $11 \mathrm{PM}$ to $11 \mathrm{AM}$, the numbers of downward power ramps is more than that of the upward. In the duration from 3:00 AM to 3:30 AM, a quick change in the numbers of power ramps happened from a high number of downward power ramps at 3:00 AM to a high number upward power ramps at 3:30 AM, this appears also in the average values of upward and downward power ramps that are presented in fig.8,9.

The average values of upward and downward power ramps in all observation times are nearly equal in the two years, but the values in 2018 are to some extent more than that in 2017 , see fig 8,9 . Hence as the average installed wind capacity increases as the average values of power ramps increase. However, when comparing the average values of power ramps with the average installed wind capacity, the two years nearly have equal ramp characteristics indicators, see fig 10, 11 .

The standard deviation values of upward and downward power ramps are shown in fig 12, 13. The curves nearly have the same values in most observation times but the values in 2018 are to some extent more than that in 2017. However, when comparing the standard deviation values of power ramps with the average installed wind capacity, the two years nearly have equal ramp characteristics indicators, see fig $14,15$.

The comparison between the average values and the standard deviation of upward and downward power ramps illustrates that the standard deviation values are greater than the average values, which exhibits the fluctuations in wind power, thus the average values of power ramps should not be used to represent the actual power ramps in wind power.

The maximum upward values of power ramps in $15 \mathrm{~min}$ interval at each observation time $t$ for years 2017, 2018 are showed in fig.16, the values of the two curves are close to each other and nearly have the same values at many observation times as in 00:00, 1:30, 2:30, 2:45, 5:30, 7:00, $7: 15,8: 00,9: 30,9: 45,11: 45,12: 30,14: 00,19: 00$ and 22:45. While the ramp characteristics indicators for maximum upward values nearly have the same values for most observation times in the two years, see fig 19. The average value of maximum upward power ramps in 2017 is 184.96 MW which represents 0.0758 by RCI While in 2018 is $224.46 \mathrm{MW}$ which represents 0.0768 by RCI. Therefore the average value of maximum upward power ramps increases with increasing the installed wind capacity but the ramp characteristics indicators approximately remain constant.

The maximum downward values of power ramps in $15 \mathrm{~min}$ interval at each observation time t for years 2017 , 2018 are showed in fig. 17, the values of the two curves are close to each other and nearly have the same values at many observation times as in 1:45, 7:15, 7:30, 11:45, 16:30, 19:00, 20:45 and 22:00. While the ramp characteristics indicators for maximum downward values nearly have the same values for most observation times in the two years, see fig 20 . The average values of maximum downward power ramps in 2017 and 2018 are 194.32 MW and 211.62 MW respectively, representing0.0797 and 0.0724 by RCI. While the average value of maximum downward power ramps increases with increasing the installed wind capacity, the ramp characteristics indicators approximately remain constant.

In fig.18, A comparison between power ramping range in $15 \mathrm{~min}$ interval at each observation time $\mathrm{t}$ for years 2017 and 2018 is shown, the ramping range in 2017 ranged from 212.11 to $793.17 \mathrm{MW}$, representing 0.087 to 0.325 by RCI, while in 2018 it ranged from 235.66 to $929.23 \mathrm{MW}$, representing 0.081 to 0.318 by RCI. While the values of ramping range in the two years are different, the RCIs are approximately equal. The average value of ramping range values in 2017 is $379.28 \mathrm{MW}$, representing0.155 by RCI, while in 2018 , it is $436.08 \mathrm{MW}$, representing0.15by RCI which is approximately the same as in 2017. Thus, while the average value and the scale of the ramping range increase 
with increasing the installed wind capacity, the RCIs of these values remain constant, see fig. 21 .

Table 1 summarizes the vertical analysis results, in which the average values in 2018 are more than that in 2017. While table 2 summarizes these values by RCI, where the average values of the two years are nearly equal. In table 2 , the average values in 2018 are to some extent less than that in 2017 except for the value of maximum upward power ramps which is to some extent more than that in 2017. In fig. 22, a comparison between the average values of vertical analysis results in the two years expressed by RCI is shown, where the average values in the two years are approximately equal. In fig.22, the average values of power ramps in the two years are nearly equal to zero at most observation times, which exhibit the fluctuations in wind power between upward and downward power ramps all times of the day, as the average numbers of upward and downward power ramps in the two years are nearly equal. However the number of downward power ramps is to some extent more than that of upward ramps, see table 1,2 .

Table 1. Vertical analysis results

\begin{tabular}{|c|c|c|c|c|c|c|}
\hline & \multicolumn{3}{|c|}{2017} & \multicolumn{3}{|c|}{2018} \\
\hline & \multicolumn{2}{|c|}{ Range } & \multicolumn{4}{|c|}{ Range } \\
\hline & From & To & Avg. & From & To & Avg. \\
\hline$\Delta p_{\max =}$ & 93.43 & 654.84 & 194.3 & 106.59 & 455.86 & 211.6 \\
\hline$\Delta p_{\max }$ & 75.34 & 444.04 & 185 & 103.89 & 736.93 & 224.46 \\
\hline$\Delta p_{\text {ang }}$ & -7.77 & 8.23 & 0.04 & -25.97 & 16 & -0.04 \\
\hline$\Delta p_{\text {ang }}$ & 18.3 & 32.4 & 23.4 & 18.89 & 42.43 & 27.29 \\
\hline$\Delta p_{a x y=0}$ & 17.5 & 31.4 & 24 & 23.13 & 37.72 & 27.76 \\
\hline$\sigma$ & 28.4 & 47.67 & 36.55 & 35.68 & 56.32 & 41.9 \\
\hline$\sigma_{\infty}$ & 18.3 & 51.8 & 27.6 & 20.87 & 51.07 & 31.02 \\
\hline$\sigma_{\infty}$ & 16.9 & 43.1 & 27.8 & 23.53 & 60.36 & 32.14 \\
\hline \multicolumn{7}{|l|}{ Ramping } \\
\hline Range & 212.1 & 793.2 & 379.3 & 235.66 & 929.23 & 436.49 \\
\hline$N_{\bullet}$ & 150 & 226 & 185 & 140 & 275 & 184 \\
\hline $\boldsymbol{N}_{\phi}$ & 139 & 215 & 180 & 90 & 225 & 181 \\
\hline
\end{tabular}

Table 2. Vertical analysis results by $\mathrm{RCI}$

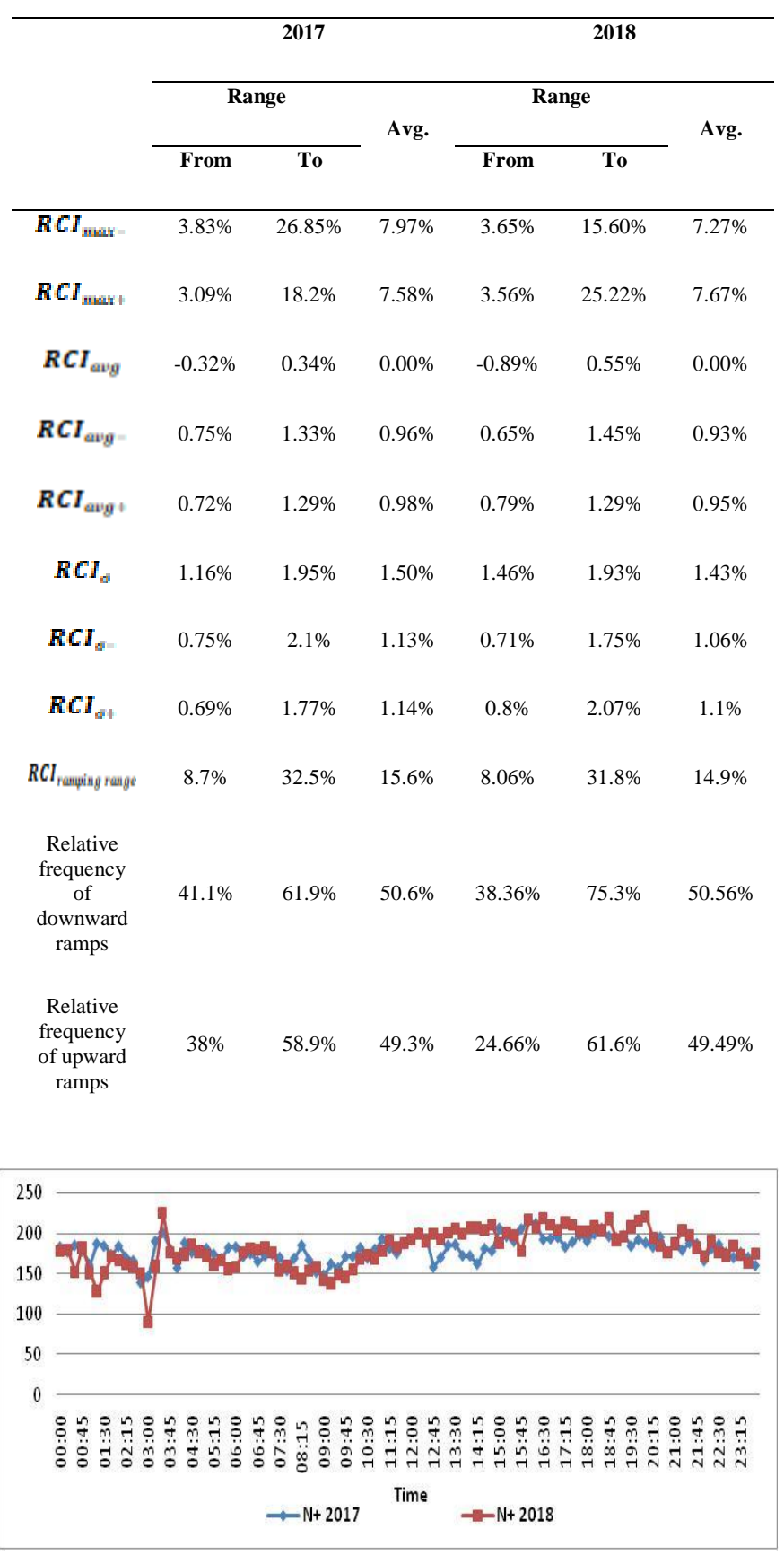

Figure 6. Comparison between numbers of upward power ramps in 15 min interval at each observation time t for the years 2017 and 2018. 


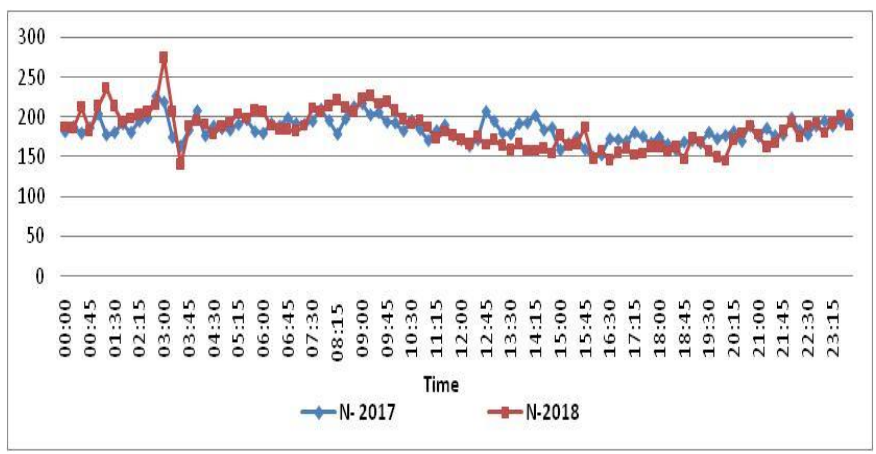

Figure 7. Comparison between numbers of downward power ramps in 15 min interval at each observation time $t$ for the years 2017 and 2018.

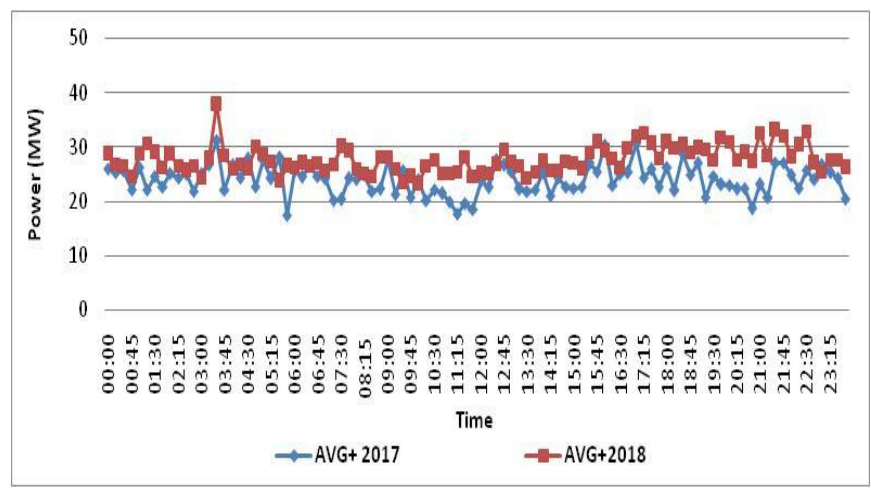

Figure 8. Comparison between average values of upward power ramps in 15 min interval at each observation time t for the years 2017 and 2018.

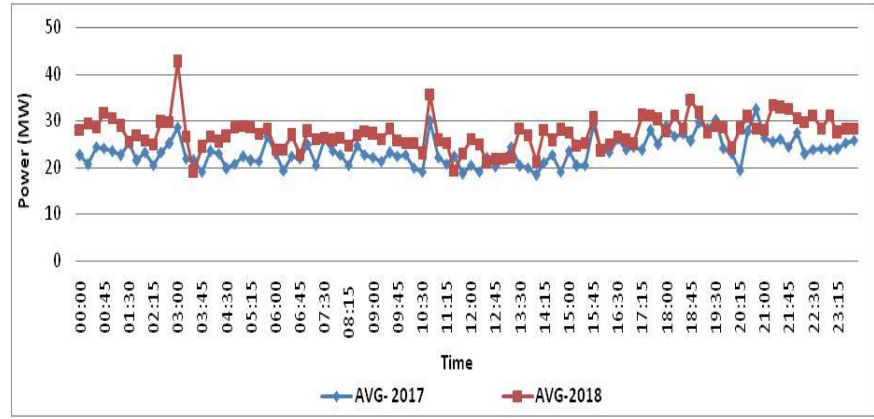

Figure 9. Comparison between average values of downward power ramps in 15 min interval at each observation time t for the years 2017 and 2018 .

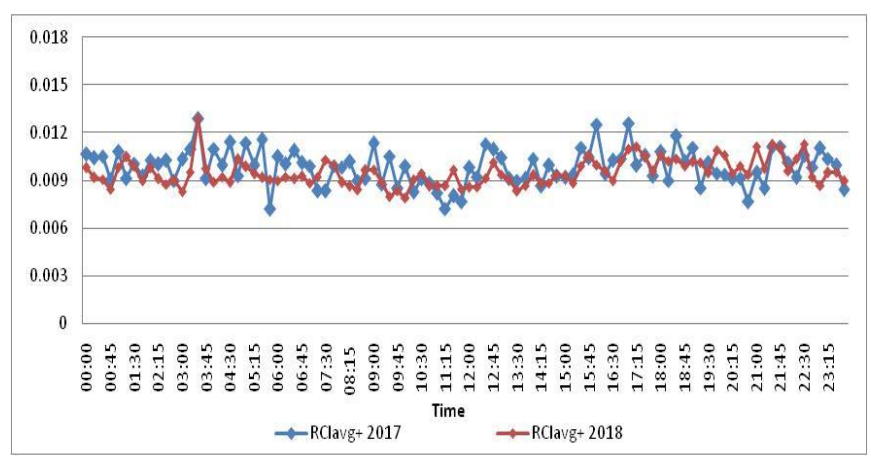

Figure 10. Comparison between $\mathrm{RCls}$ for the average of upward power ramps in 15 min interval at each observation time $t$ for the years 2017 and 2018.

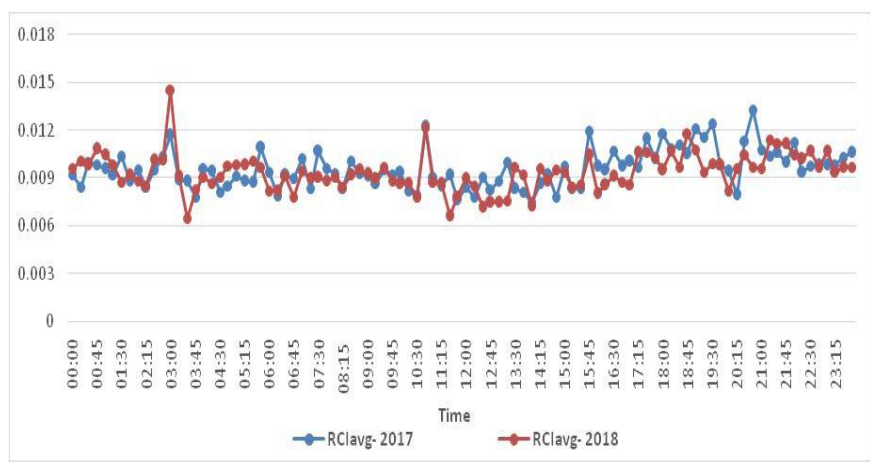

Figure 11. Comparison between RCls for the average of downward power ramps in 15 min interval at each observation time $\mathrm{t}$ for the years 2017 and 2018 .

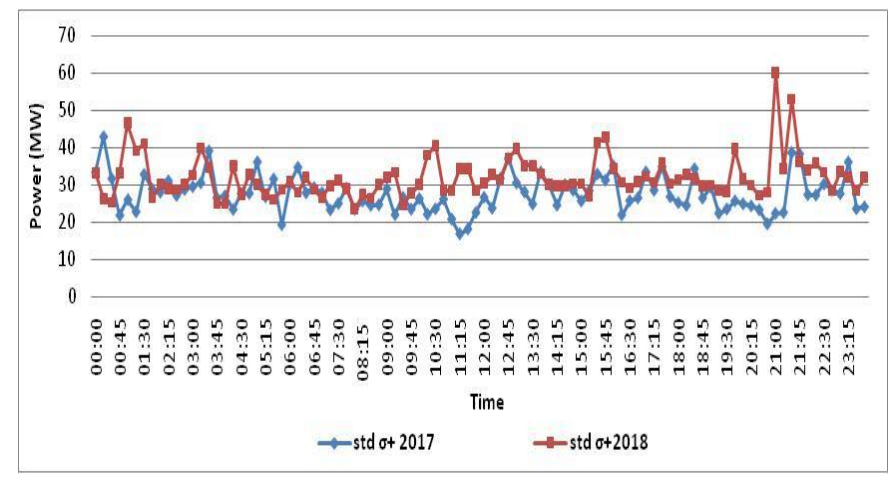

Figure 12. Comparison between standard deviation values of upward power ramps in $15 \mathrm{~min}$ interval at each observation time t for the years 2017 and 2018. 


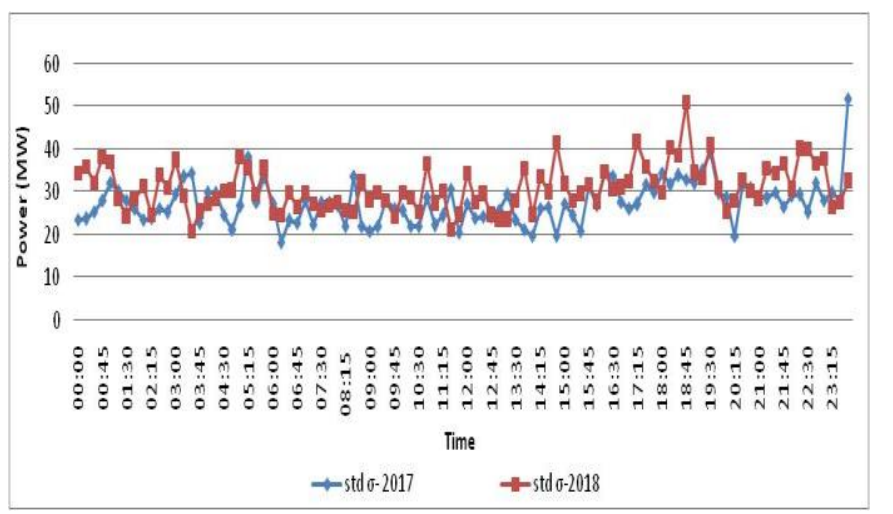

Figure 13. Comparison between standard deviation values of downward power ramps in 15 min interval at each observation time $t$ for the years 2017 and 2018.

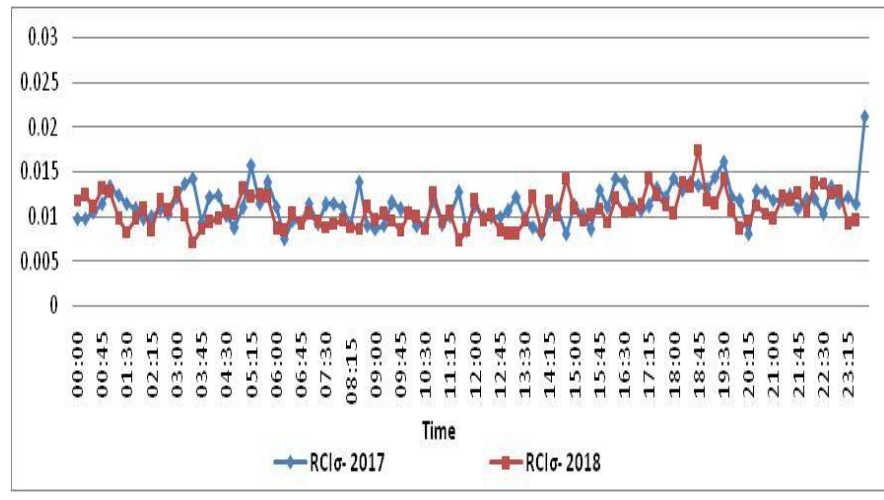

Figure 14. Comparison between RCls for the standard deviation of downward power ramps in 15 min interval at each observation time $t$ for the years 2017 and 2018.

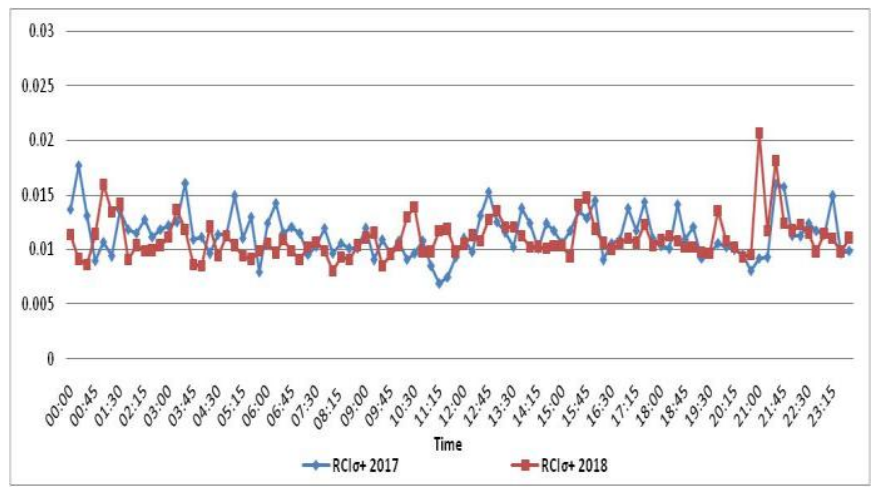

Figure 15. Comparison between RCls for the standard deviation of upward power ramps in 15 min interval at each observation time $t$ for the years 2017 and 2018.

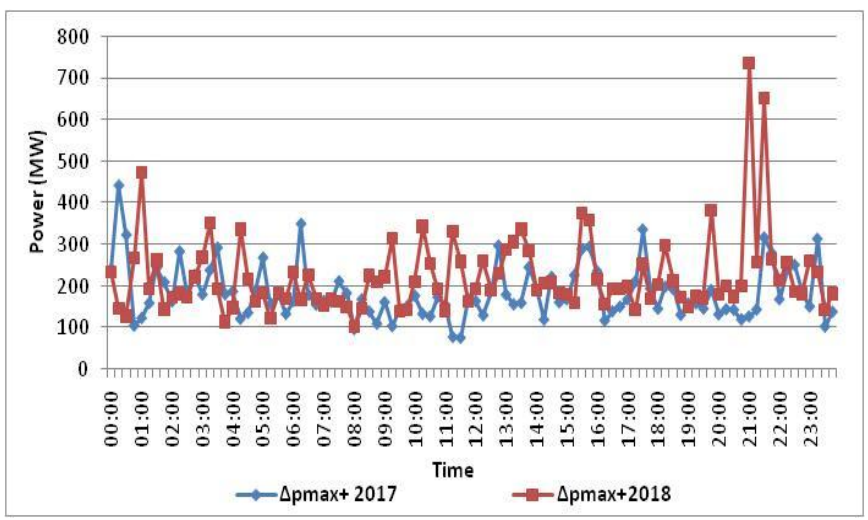

Figure 16. Comparison between maximum values of upward power ramps in 15 min interval at each observation time t for the years 2017 and 2018.

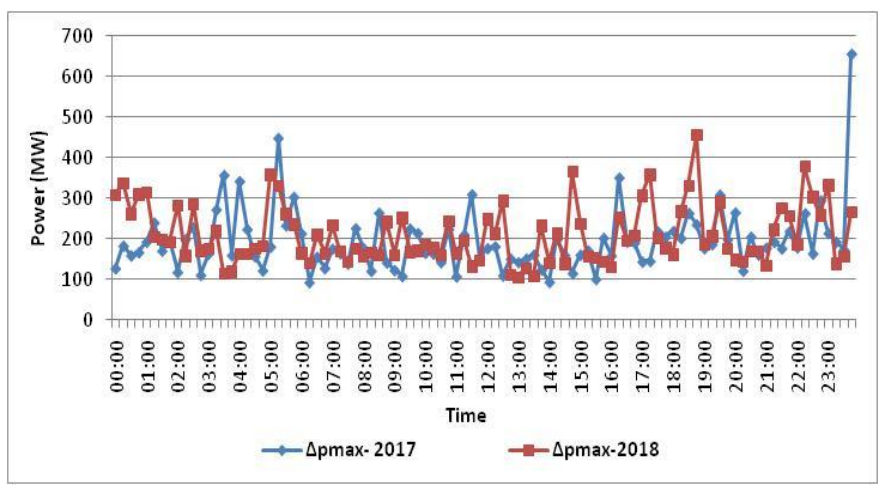

Figure 17. Comparison between maximum values of downward power ramps in 15 min interval at each observation time t for the years 2017 and 2018.

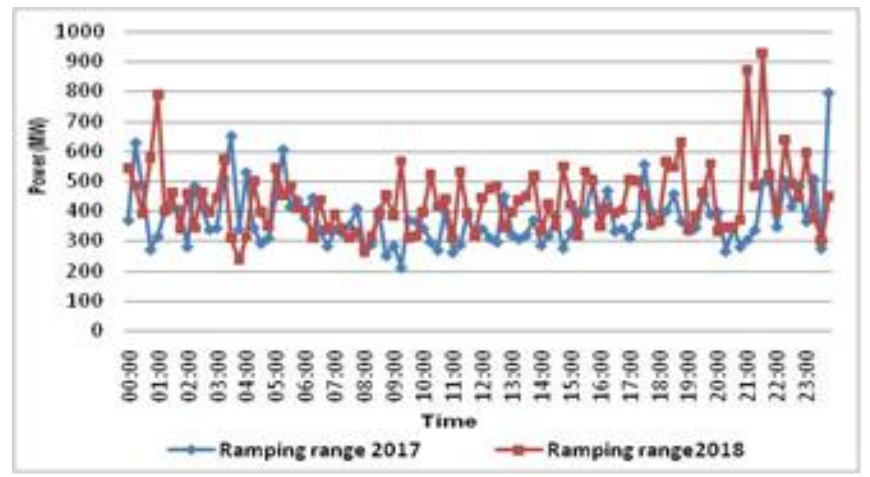

Figure 18. Comparison between ramping range values of power ramps in 15 min interval at each observation time $t$ for the years 2017 and 2018. 


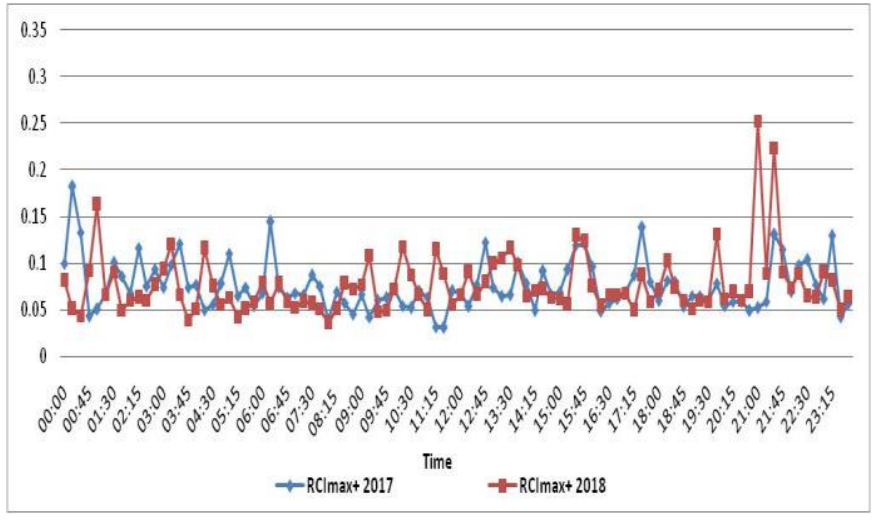

Figure 19. Comparison between $\mathrm{RCl}$ for maximum values of upward power ramps in 15 min interval at each observation time $t$ for the years 2017 and 2018.

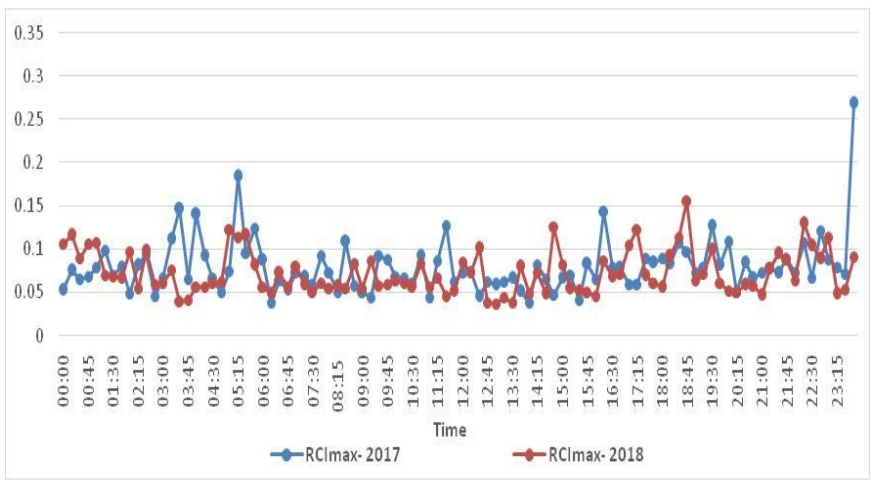

Figure 20. Comparison between $\mathrm{RCl}$ for maximum values of downward power ramps in 15 min interval at each observation time $\mathrm{t}$ for the years 2017 and 2018.

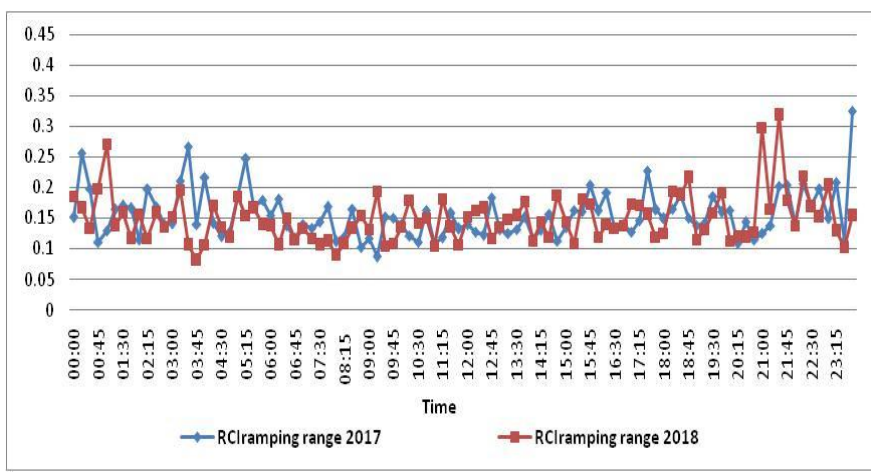

Figure 21. Comparison between $\mathrm{RCl}$ for ramping range values of power ramps in 15 min interval at each observation time t for the years 2017 and 2018.

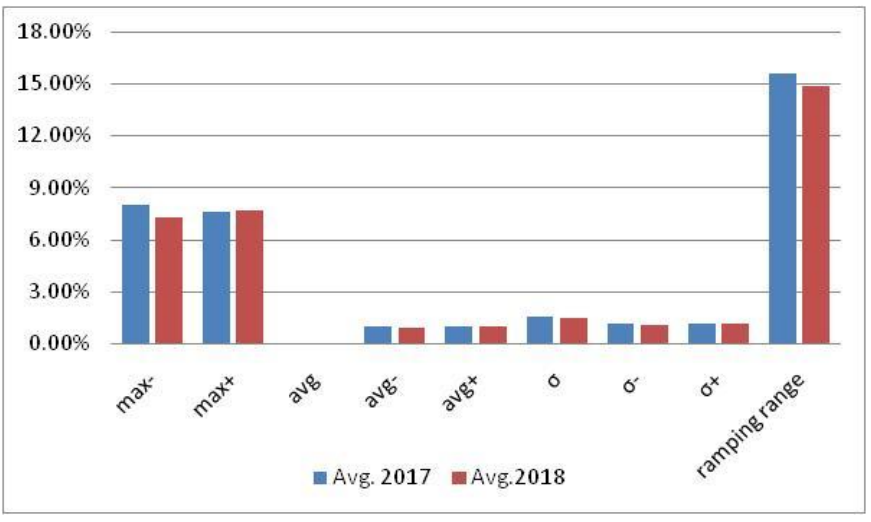

Figure 22. Average values of vertical analysis results by $\mathrm{RCl}$ for the years 2017 and 2018

\subsection{Horizontal analysis results and discussion}

In fig.23 and fig.24, the numbers of upward and downward power ramps for each month are presented, where numbers of both ramp types are nearly the same in all months in the two years, which exhibits the continuous variation of wind power between upward and downward power ramps.

In fig. 25 and fig.26, the average values of upward and downward power ramps for each month are presented. The average values of both ramp types in 2018 are greater than that in 2017 for most months, while the RCIs for most months in the two years nearly equal except January and July, see fig. 27, 28.

The comparison between the average values and the standard deviation of both ramp types illustrates that their values are very close, also the standard deviation values are to some extent greater than that of the average values, see table 2. The standard deviation values of the two years are very close when calculating the RCI for these values, see fig. 31, 32 .

In fig. 33, 37, the comparison between the maximum values of upward power ramps in the two years illustrates that the values are very close in most months except March, July and August. The average values of maximum upward power ramps in 2017 and 2018 are 283.21 and $346.49 \mathrm{MW}$ respectively, representing $11.6 \%$ and $11.9 \%$ by RCI, which are approximately equal.

The comparison between the maximum values of downward power ramps for the two years illustrates that the values are very close in most months except January, February and June, see fig.34,38. The average values of maximum downward power ramps in 2017 and 2018 are 317.84 and 316.62 MW respectively, representing $13 \%$ and $11 \%$ by RCI.

In fig. 35,36 , a comparison between the power ramping range in 15min interval for each month for years 2017 and 2018 is shown, the power ramping range in 2017 ranged from 434.86 to $969.78 \mathrm{MW}$, representing $17.83 \%$ to 
$39.76 \%$, respectively, of the average installed wind capacity in that year, while in 2018 ranged from 362.21 to 1074.92 MW, representing $12.4 \%$ to $36.79 \%$, respectively, of the average installed wind capacity. The average values of power ramping range in 2017 and 2018 are 601.04 and $663.11 \mathrm{MW}$, representing $24.6 \%$ and $22.7 \%$ of the average installed wind capacity respectively, which are approximately equal.

Table 3 summarizes the horizontal analysis results, in which the average values in 2018 are more than that in 2017 except that of maximum downward power ramps. While table 4 summarizes these values by RCI, where the average values of the two years are nearly equal. In table 4 , the average values in 2018 are to some extent less than that in 2017 except for the value of maximum upward power ramps which is to some extent more than that in 2017. In fig. 39, a comparison between the average values of horizontal analysis results in the two years expressed by RCI is shown, where the average values in the two years are approximately equal. The average numbers of upward and downward power ramps in the two years are nearly equal; however, the number of downward power ramps is more than that of upward ramps.

Table 3. Horizontal analysis results

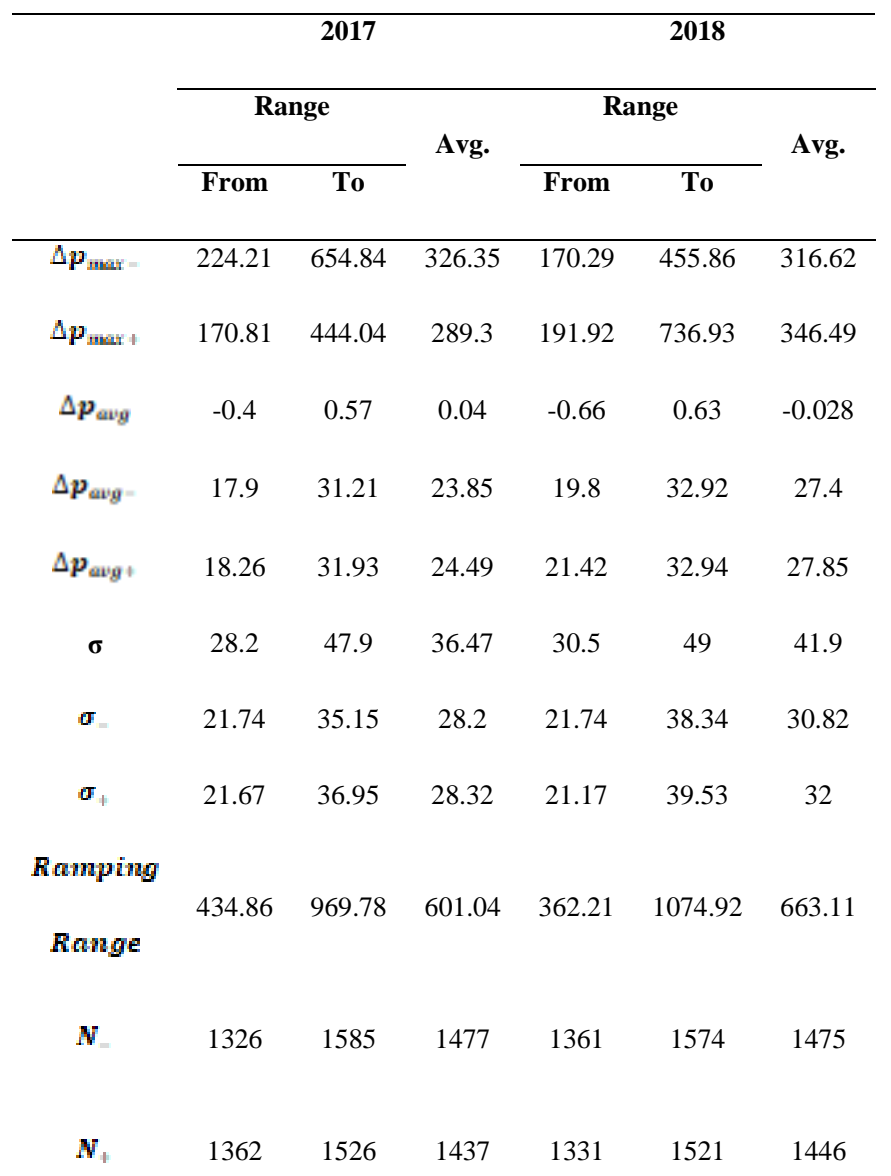

Table 4. Horizontal analysis results by $\mathrm{RCI}$

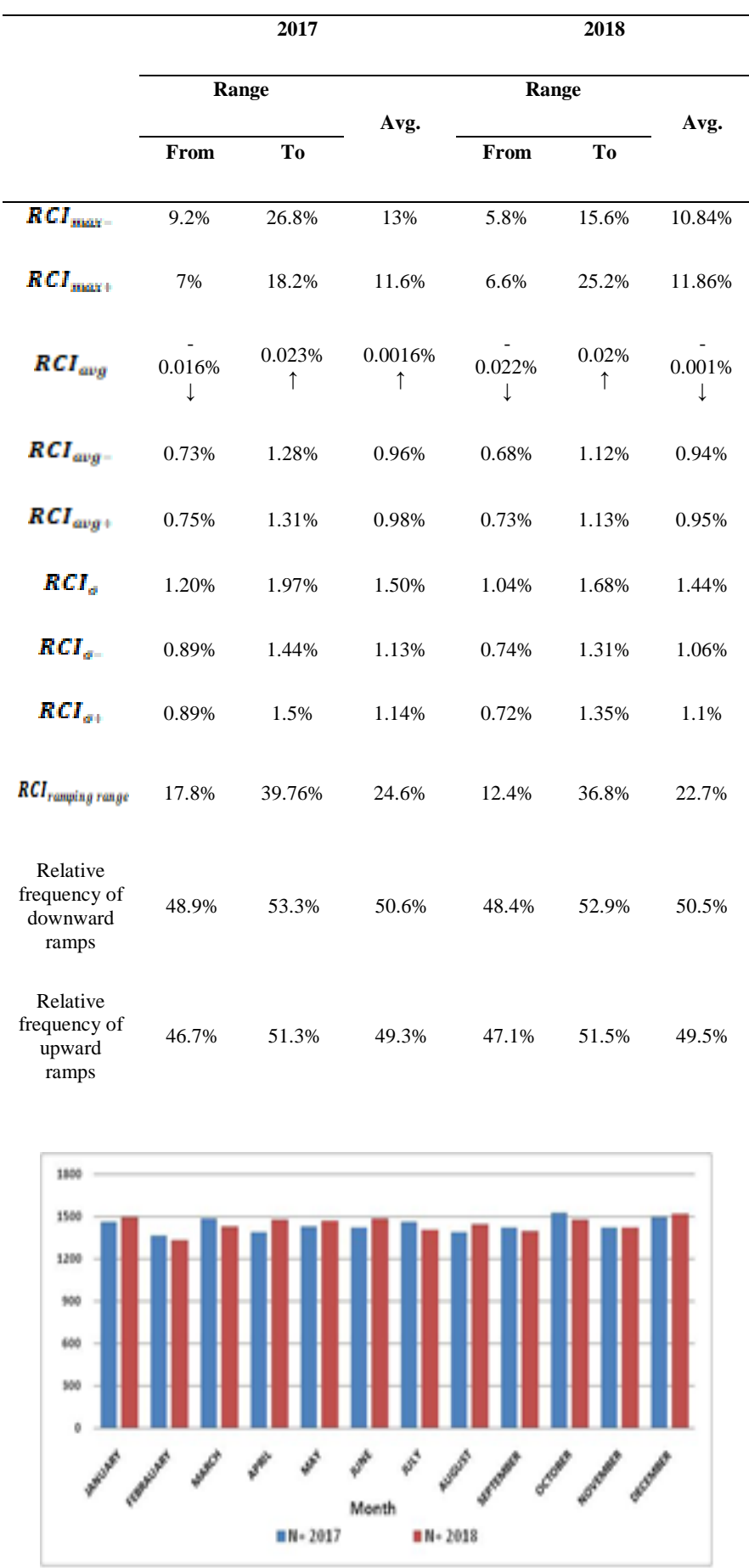

Figure 23. Comparison between numbers of upward power ramps in 15 min interval for each month for the years 2017 and 2018. 


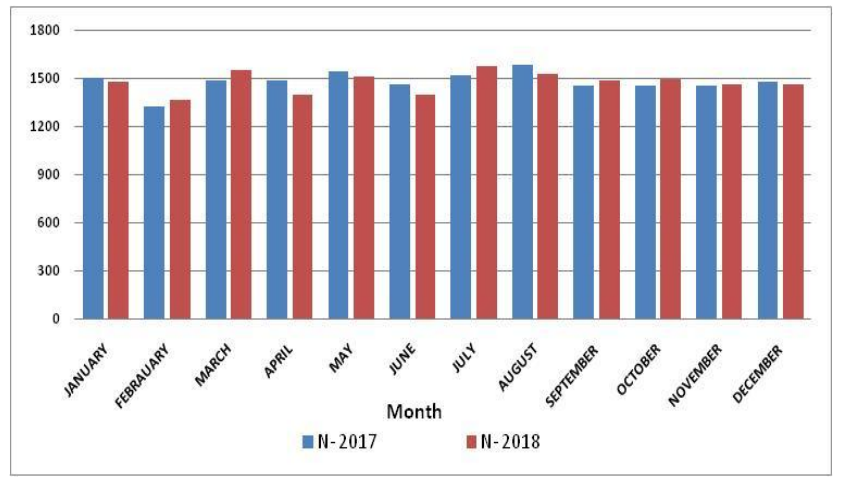

Figure 24. Comparison between numbers of downward power ramps in $15 \mathrm{~min}$ interval for each month for the years 2017 and 2018.

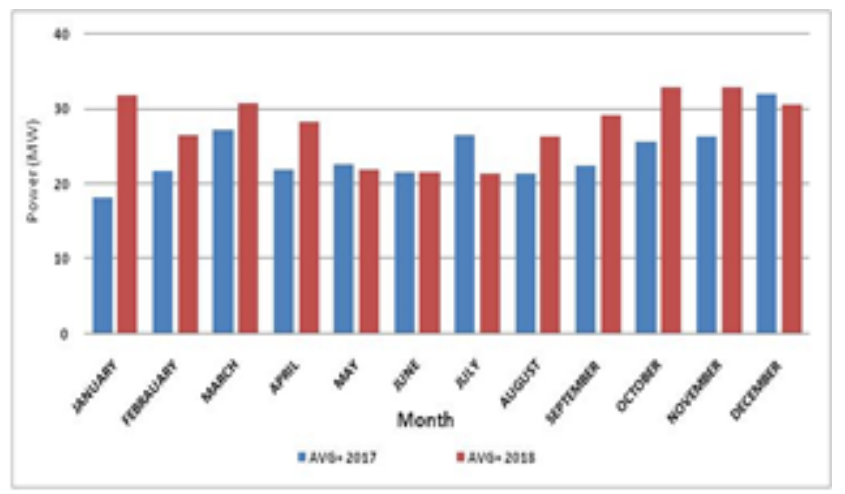

Figure 25. Comparison between average values of upward power ramps in 15 min interval for each month for years 2017 and 2018.

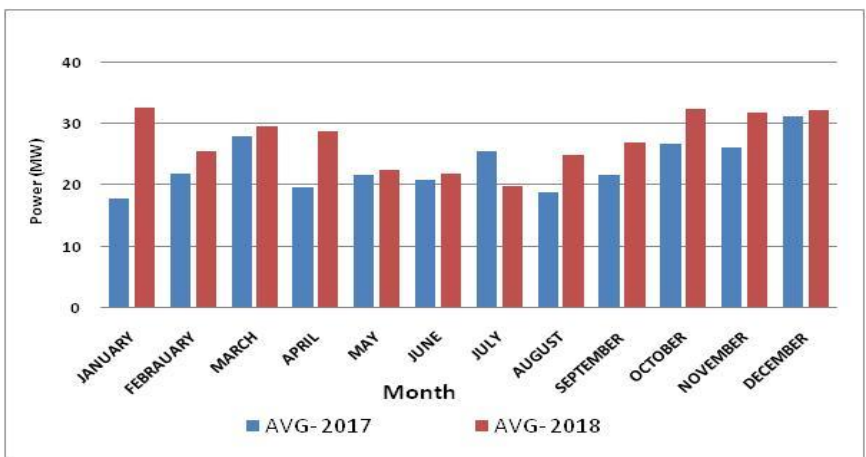

Figure 26. Comparison between average values of downward power ramps in 15 min interval for each month for years 2017 and 2018.

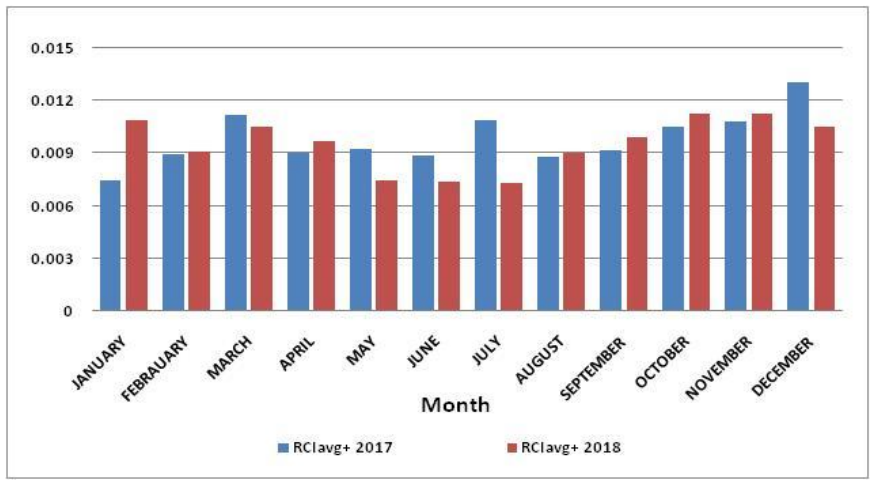

Figure 27. Comparison between $\mathrm{RCls}$ for the average values of upward power ramps in 15 min interval for each month for years 2017 and 2018.

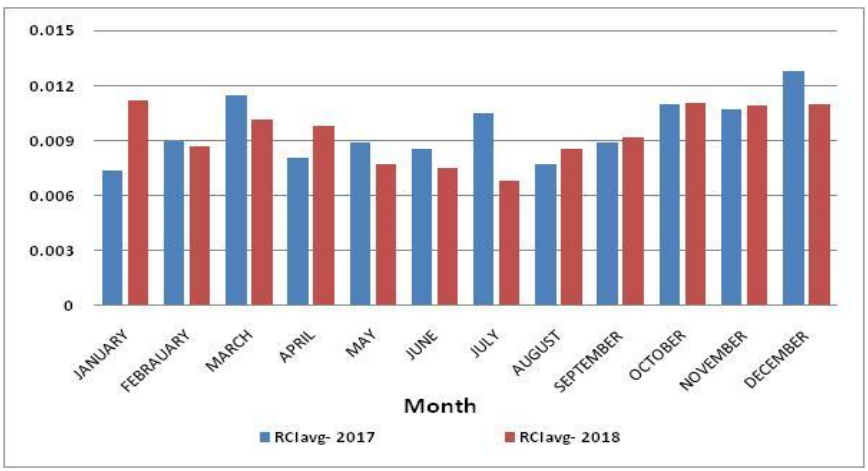

Figure 28. Comparison between $\mathrm{RCls}$ for the average values of downward power ramps in 15 min interval for each month for the years 2017 and 2018.

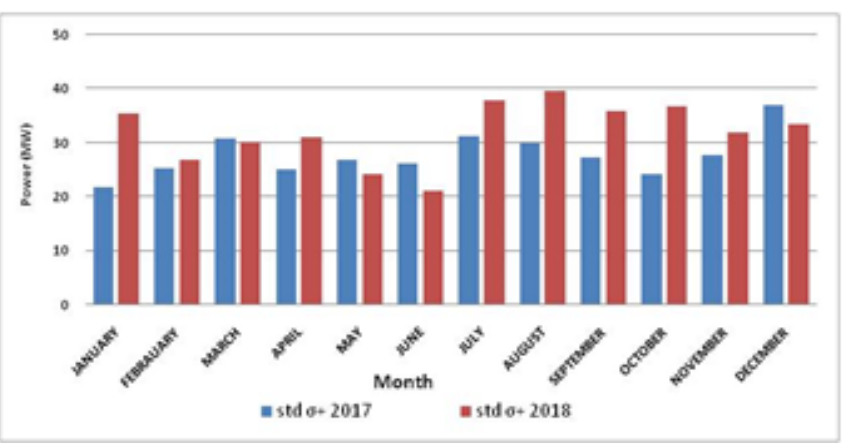

Figure 29. Comparison between standard deviation values of upward power ramps in 15 min interval for each month for years 2017 and 2018. 


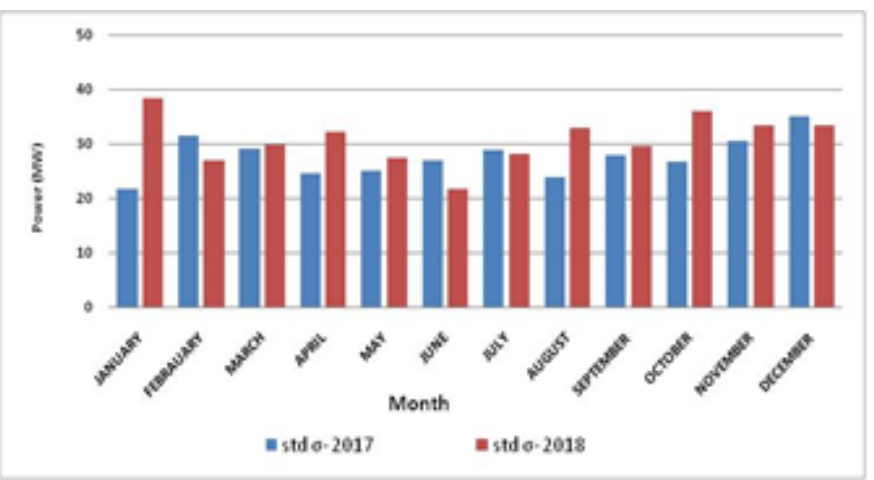

Figure 30. Comparison between standard deviation values of downward power ramps in 15 min interval for each month for years 2017 and 2018.

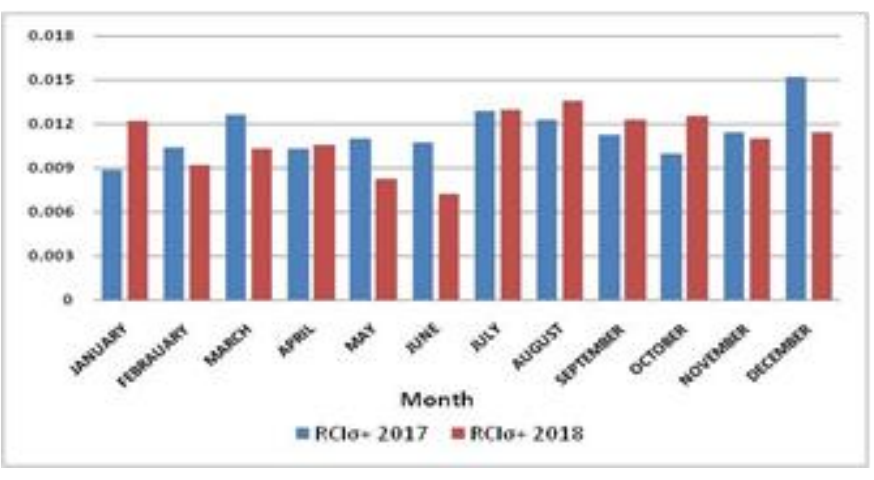

Figure 31. Comparison between RCls for the standard deviation of upward power ramps in 15 min interval for each month for years 2017 and 2018.

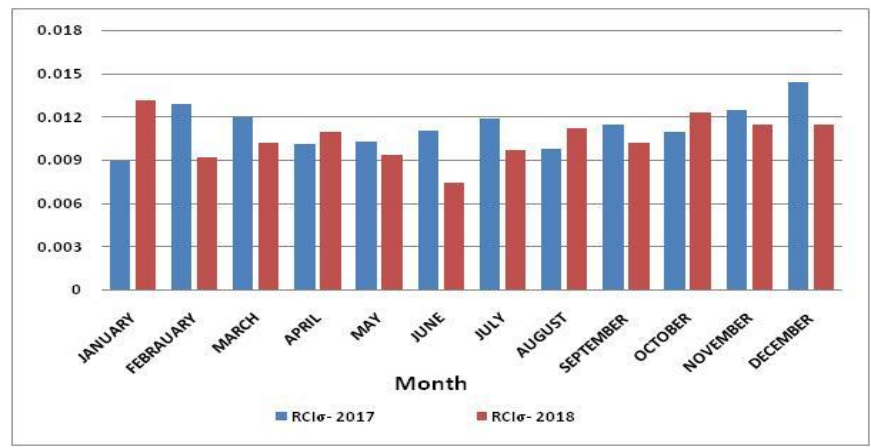

Figure 32. Comparison between RCls for the standard deviation of downward power ramps in 15 min interval for each month for years 2017 and 2018.

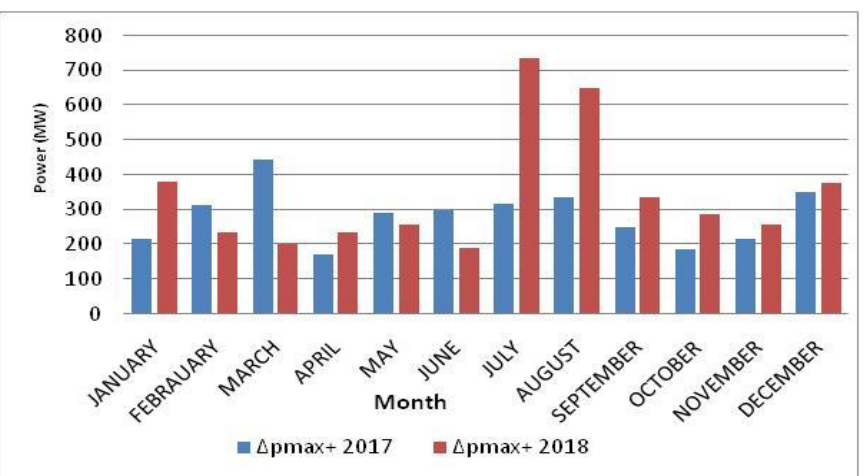

Figure 33. Comparison between maximum values of upward power ramps in 15 min interval for each month for years 2017 and 2018.

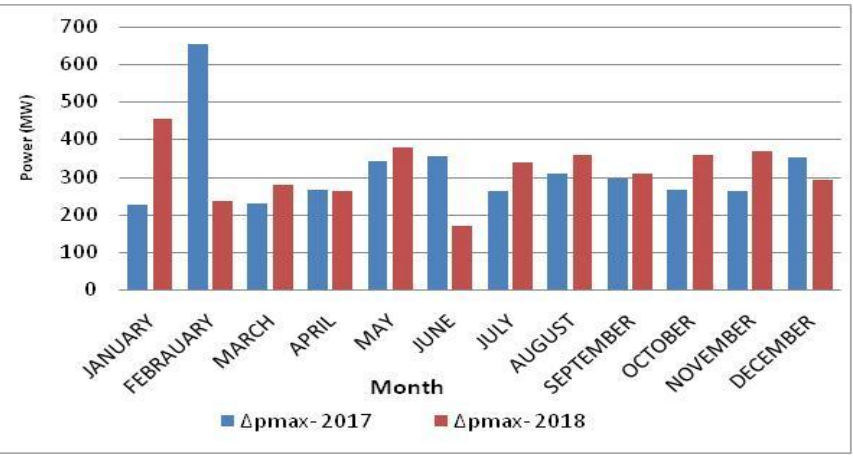

Figure 34. Comparison between maximum values of downward power ramps in 15 min interval for each month for years 2017 and 2018.

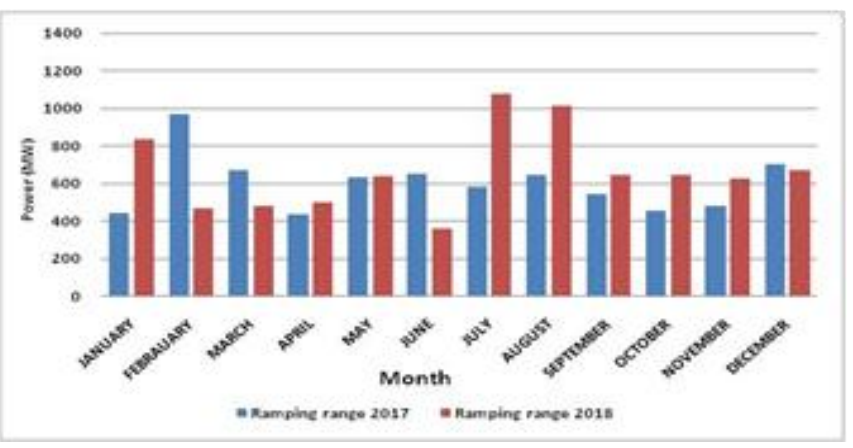

Figure 35. Comparison between the ramping range of power ramps in 15 min interval for each month for years 2017 and 2018. 


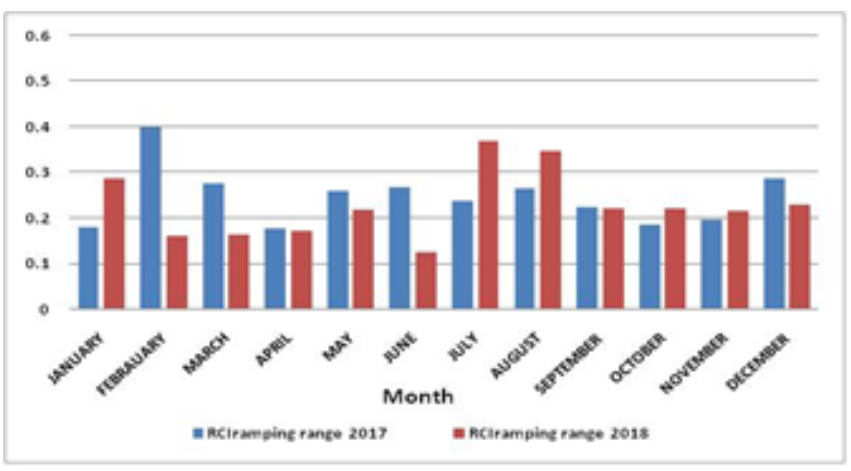

Figure 36. Comparison between $\mathrm{RCl}$ for ramping range of power ramps in 15 min interval for each month for years 2017 and 2018.

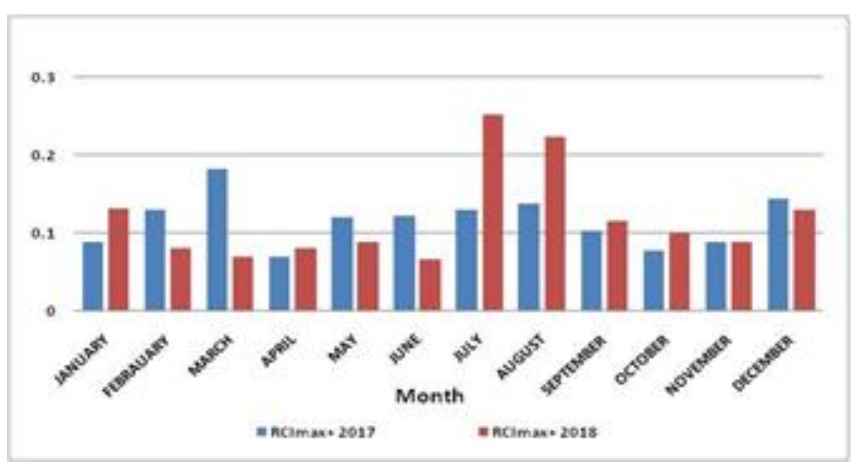

Figure 37. Comparison between $\mathrm{RCl}$ for maximum values of upward power ramps in 15 min interval for each month for years 2017 and 2018.

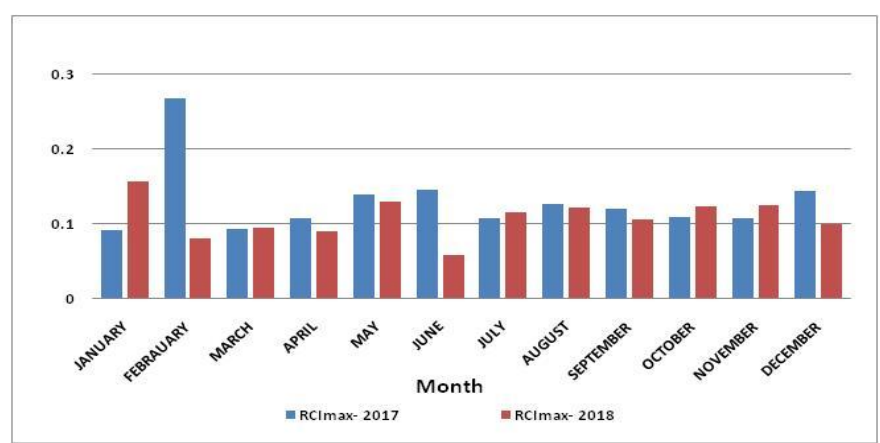

Figure 38. Comparison between $\mathrm{RCl}$ for maximum values of downward power ramps in 15 min interval for each month for years 2017 and 2018

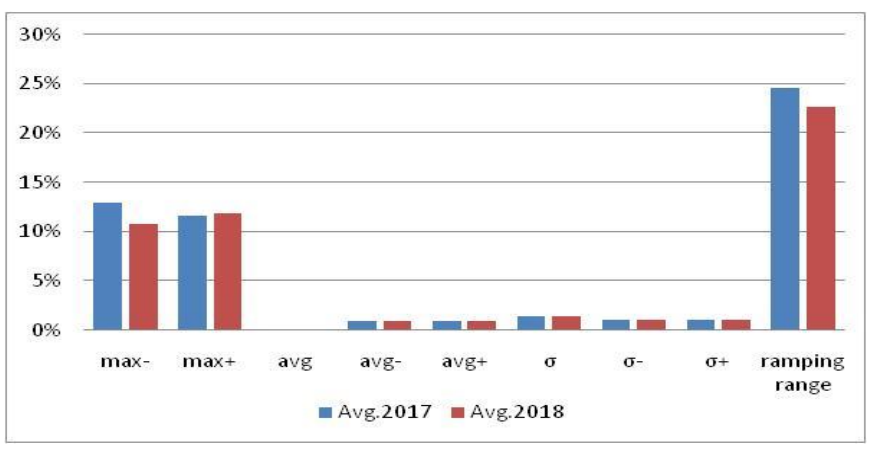

Figure 39. Average values of horizontal analysis results by $\mathrm{RCl}$

\section{Conclusion}

Flexibility metrics in power systems are ranged from simplicity to complexity according to the constraints that were taken into consideration during evolving the metric. Several assumptions were taken into considerations in these metrics that depend on whether the metric is used in operation or planning stages. Although several flexibility metrics and evaluation techniques existed, up-till-now there is no flexibility metric that taken as a standard. Consequently, metrics are still developing. The flexibility metrics studies illustrated that the important factor in power system flexibility is the power ramps in VRG. While an improvement in wind power forecasting has been achieved, the percentage forecasting error is still relatively high. Therefore, as the VRG shares increases in the system, as the forecasting error impacts the balance between generation and consumption. Consequently, the power system operators should have more information about the behaviour of VRG power ramps in the system, which includes the scale of power ramps as well as the expected times at which high power ramps are most likely to occur, these information complements that forecasted to achieve flexibility and reliability in the power system.

The paper proposed an analysis method based on extracting information from the historical databases to clarify the power ramping features of VRG in a power system. These analysis procedures can also be used to analyze the power ramps in load or net load and not limited to a specific power system, in which the historical data of the power-time curve is analyzed in two directions which are vertical and horizontal. In the vertical direction, the historical power ramps during the studied time interval $\Delta \mathrm{t}$ for each observation time $(\mathrm{t})$ in the power signal time series is studied separately in detail. While in the horizontal direction, the daily historical readings of power ramps occurred during the studied time interval $\Delta t$ throughout each day are studied to get information about the power ramping behaviour in certain weeks, months, seasons or years. The ramp characteristic indicators are proposed for comparing 
the ramping behaviour of VRG in different years or between different countries. The analysis procedures are demonstrated by analyzing the output power of aggregated Belgium's wind farms in the years 2017, 2018 and comparing the results of the two years. The results obtained by the two analysis directions can be summarized in the following points:

$>$ The standard deviation is very high compared to the average power ramp.

$>$ The average values of upward and downward power ramps nearly equal to that of standard deviation, so the average value of wind power ramps should not be utilized to represent the actual power ramps in wind power.

$>$ Although the changes in wind power output are difficult to be predicted with a high degree of accuracy, it is possible to determine the extent of these changes. Even if the extent of these changes has changed with increasing the installed wind capacity, the changes nearly have a fixed percentage when comparing it with the average value of installed wind capacity during the year, see Fig. 22, 39.

\section{References}

[1] M. S. Eltohamy and M. S. A. Moteleb, "Overview of Power System Flexibility Options with Increasing Variable Renewable Generations," in In 6th international conference on advanced control circuits and systems accs019, 17-19 November, Hurghada, Egypt, organized by Electronics Research Institute (ERI), 2019, doi: 10.1109/ACCS-PEIT48329.2019.9062836.

[2] Z. Lu, H. Li, and Y. Qiao, "Probabilistic Flexibility Evaluation for Power System Planning Considering Its Association With Renewable Power Curtailment," IEEE Trans. Power Syst., vol. 33, no. 3, pp. 3285-3295, 2018, doi: 10.1109/TPWRS.2018.2810091.

[3] H. Nosair and F. Bouffard, "Energy-Centric Flexibility Management in Power Systems," IEEE Trans. Power Syst., vol. 31, no. 6, pp. 5071-5081, 2016, doi: 10.1109/TPWRS.2015.2512990.

[4] J. Cochran et al., "Flexibility in 21st Century Power Systems," 21st Century Power Partnersh., vol. May, p. 14, 2014, doi: 10.2172/1130630.

[5] M. A. Bucher, S. Delikaraoglou, K. Heussen, P. Pinson, and G. Andersson, "On quantification of flexibility in power systems," 2015 IEEE Eindhoven PowerTech, PowerTech 2015, pp. 1-6, 2015, doi: 10.1109/PTC.2015.7232514.

[6] E. Lannoye, D. Flynn, and M. O'Malley, "Assessment of power system flexibility: A high-level approach," IEEE Power Energy Soc. Gen. Meet., 2012, doi: 10.1109/PESGM.2012.6345435.

[7] A. Ulbig and G. Andersson, "Analyzing operational flexibility of electric power systems," Int. J. Electr. Power Energy Syst., vol. 72, pp. 155-164, 2015, doi: 10.1016/j.ijepes.2015.02.028.

[8] H. Nosair and F. Bouffard, "Flexibility Envelopes for Power System Operational Planning," IEEE Trans. Sustain. Energy, vol. 6, no. 3, pp. 800-809, 2015, doi: 10.1109/TSTE.2015.2410760.

[9] H. Nosair and F. Bouffard, "Reconstructing Operating
Reserve: Flexibility for Sustainable Power Systems," IEEE Trans. Sustain. Energy, vol. 6, no. 4, pp. 16241637, 2015, doi: 10.1109/TSTE.2015.2462318

[10] J. Ma, "Evaluating and Planning Flexibility in a Sustainable Power System with Large Wind Penetration," p. 202, 2012.

[11] V. Oree and S. Z. Sayed Hassen, “A composite metric for assessing flexibility available in conventional generators of power systems," Appl. Energy, vol. 177, pp. 683-691, 2016, doi: 10.1016/j.apenergy.2016.05.138.

[12] J. Zhao, T. Zheng, and E. Litvinov, "A unified framework for defining and measuring flexibility in power system," IEEE Trans. Power Syst., vol. 31, no. 1, pp. 339-347, 2016, doi: 10.1109/TPWRS.2015.2390038.

[13] Eirgrid, "Ensuring a Secure, Reliable and Efficient Power System in a Changing Environment June 2011," no. June, p. 73, 2011.

[14] Eirgrid, "Delivering a Secure Sustainable Electricity System (DS3)," 2011, [Online]. Available: http://www.eirgrid.com/operations/ds3/.

[15] N. Menemenlis, M. Huneault, and A. Robitaille, "Thoughts on power system flexibility quantification for the short-term horizon," IEEE Power Energy Soc. Gen. Meet., pp. 1-8, 2011, doi: 10.1109/PES.2011.6039617.

[16] D. Kazmer, D. Hatch, L. Zhu, C. Roser, and D. Kapoor, "Definition and Application of a Process Flexibility Index," J. Manuf. Sci. Eng., vol. 125, no. 1, p. 164, 2003, doi: $10.1115 / 1.1536174$.

[17] E. Lannoye, D. Flynn, and M. O'Malley, "Evaluation of power system flexibility," IEEE Trans. Power Syst., vol. 27, no. 2, pp. 922-931, 2012, doi: 10.1109/TPWRS.2011.2177280.

[18] E. Lannoye, A. O. Mahoney, and A. Muireann, "Econometric Analysis of Flexibility Rewards in Electricity Markets," Int. Ser. Oper. Res. Manag. Sci., vol. 153, pp. 1-26, 2010, doi: 10.1007/978-1-4419-7421-1_1.

[19] E. Lannoye, D. Flynn, and M. O'Malley, "Transmission, variable generation, and power system flexibility," IEEE Trans. Power Syst., vol. 30, no. 1, pp. 57-66, 2015, doi: 10.1109/TPWRS.2014.2321793.

[20] M. Saber, F. M. A. Ghali, and E. E. A. Elzahab, "The effect of DG penetration on short circuit currents level," in ACCS/PEIT 2017 - 2017 Intl Conf on Advanced Control Circuits Systems and 2017 Intl Conf on New Paradigms in Electronics and Information Technology, 2018, vol. 2018Febru, doi: 10.1109/ACCS-PEIT.2017.8303054.

[21] M. Huber, D. Dimkova, and T. Hamacher, "Integration of wind and solar power in Europe: Assessment of flexibility requirements," Energy, vol. 69, pp. 236-246, 2014, doi: 10.1016/j.energy.2014.02.109.

[22] E. Gomez-lazaro et al., "“Flexibility chart: Evaluation on diversity of flexibility in various areas.," in 12th International Workshop on Large-Scale Integration of Wind Power into Power Systems as well as on Transmission Networks for Offshore Wind Farms, WIW13. Energynautics GmbH, 2013., 2013.

[23] J. Ma, D. S. Kirschen, R. Belhomme, and V. Silva, "Optimizing the flexibility of a portfolio of generating plants," 17th Power Syst. Comput. Conf. PSCC 2011, no. January, 2011, doi: 10.13140/2.1.3535.9048.

[24] S. Sreekumar, K. C. Sharma, and R. Bhakar, "Gumbel copula based multi interval ramp product for power system flexibility enhancement," Int. J. Electr. Power Energy Syst., vol. 112, no. June 2018, pp. 417-427, 2019, doi: 10.1016/j.ijepes.2019.05.018.

[25] C. Wang, P. Bao-Sen Luh, and N. Navid, "Ramp 
Requirement Design for Reliable and Efficient Integration of Renewable Energy," IEEE Trans. Power Syst., vol. 32, no. $\quad 1, \quad$ pp. 562-571, 2017, doi: 10.1109/TPWRS.2016.2555855.

[26] M. Heleno, R. Soares, J. Sumaili, R. J. Bessa, L. Seca, and M. A. Matos, "Estimation of the flexibility range in the transmission-distribution boundary," 2015 IEEE Eindhoven PowerTech, PowerTech 2015, 2015, doi: 10.1109/PTC.2015.7232524.

[27] J. Silva, J. Sumaili, R. J. Bessa, L. Seca, M. Matos, and V. Miranda, "The challenges of estimating the impact of distributed energy resources flexibility on the TSO/DSO boundary node operating points," Comput. Oper. Res., vol. 96, pp. 294-304, 2018, doi: 10.1016/j.cor.2017.06.004.

[28] H. Chandler, Harnessing variable renewables: A guide to the balancing challenge. IEA, 2011.

[29] OECD. Publishing and I. E. Agency., "Harnessing variable renewables: a guide to the balancing challenge," Organ. Econ. Co-operation Dev., 2011.

[30] I. Renewable and E. Agency, "Power system flexibility for the energy transition,Part 1: Overview for policy makers," 2018. doi: 10.13140/RG.2.2.11150.61768.

[31] V. Oree, S. Z. Sayed Hassen, and P. J. Fleming, "Generation expansion planning optimisation with renewable energy integration: A review," Renew. Sustain. Energy Rev., vol. 69, no. December 2016, pp. 790-803, 2017, doi: 10.1016/j.rser.2016.11.120.

[32] EPRI, "Metrics for Quantifying Flexibility in Power System Planning," p. 16, 2014.

[33] R. Billinton and M. Fotuhi-Firuzabad, "A Basic Framework for Generating System Operating Health Analysis," IEEE Trans. Power Syst., vol. 9, no. 3, pp. 1610-1617, 1994, doi: 10.1109/59.336097.

[34] J. Hargreaves, E. K. Hart, R. Jones, and A. Olson, "REFLEX: An Adapted Production Simulation Methodology for Flexible Capacity Planning," IEEE Trans. Power Syst., vol. 30, no. 3, pp. 1306-1315, 2015, doi: 10.1109/TPWRS.2014.2351235.

[35] E. Lannoye et al., "Integration of variable generation: Capacity value and evaluation of flexibility," IEEE PES Gen. Meet. PES 2010, pp. 1-6, 2010, doi: 10.1109/PES.2010.5589889.

[36] M. Milligan and K. Porter, "Determining the Capacity Value of Wind: An Updated Survey of Methods and Implementation - Conference paper to be presented at WindPower 2008," 2008.

[37] M. R. Milligan and K. Porter, "Determining the Capacity Value of Wind: A Survey of Methods and Implementation," 2005.

[38] M. A. Bucher, S. Chatzivasileiadis, and G. Andersson, "Managing Flexibility in Multi-Area Power Systems," IEEE Trans. Power Syst., vol. 31, no. 2, pp. 1218-1226, 2016, doi: 10.1109/TPWRS.2015.2413387.

[39] A. A. Thatte and L. Xie, "A metric and market construct of inter-temporal flexibility in time-coupled economic dispatch," IEEE Trans. Power Syst., vol. 31, no. 5, pp. 3437-3446, 2016, doi: 10.1109/TPWRS.2015.2495118.

[40] B. Zhang and M. Kezunovic, "Impact on Power System Flexibility by Electric Vehicle Participation in Ramp Market," IEEE Trans. Smart Grid, vol. 7, no. 3, pp. 12851294, 2016, doi: 10.1109/TSG.2015.2437911.

[41] "Elia, Belgium's electricity transmission system operator." http://www.elia.be/en/grid-data/powergeneration/wind-power (accessed Feb. 15, 2019). 